\title{
Extracting physiological information in experimental biology via Eulerian video magnification
}

\author{
Henrik Lauridsen ${ }^{1,2^{*}}$ (D), Selina Gonzales ${ }^{1,3}$, Daniela Hedwig ${ }^{4}$, Kathryn L. Perrin ${ }^{5,6}$, Catherine J. A. Williams ${ }^{5,7}$,
} Peter H. Wrege ${ }^{4}$, Mads F. Bertelsen ${ }^{5,6}$, Michael Pedersen ${ }^{2}$ and Jonathan T. Butcher ${ }^{1}$

\begin{abstract}
Background: Videographic material of animals can contain inapparent signals, such as color changes or motion that hold information about physiological functions, such as heart and respiration rate, pulse wave velocity, and vocalization. Eulerian video magnification allows the enhancement of such signals to enable their detection. The purpose of this study is to demonstrate how signals relevant to experimental physiology can be extracted from non-contact videographic material of animals.

Results: We applied Eulerian video magnification to detect physiological signals in a range of experimental models and in captive and free ranging wildlife. Neotenic Mexican axolotls were studied to demonstrate the extraction of heart rate signal of non-embryonic animals from dedicated videographic material. Heart rate could be acquired both in single and multiple animal setups of leucistic and normally colored animals under different physiological conditions (resting, exercised, or anesthetized) using a wide range of video qualities. Pulse wave velocity could also be measured in the low blood pressure system of the axolotl as well as in the high-pressure system of the human being. Heart rate extraction was also possible from videos of conscious, unconstrained zebrafish and from nondedicated videographic material of sand lizard and giraffe. This technique also allowed for heart rate detection in embryonic chickens in ovo through the eggshell and in embryonic mice in utero and could be used as a gating signal to acquire two-phase volumetric micro-CT data of the beating embryonic chicken heart. Additionally, Eulerian video magnification was used to demonstrate how vocalization-induced vibrations can be detected in infrasoundproducing Asian elephants.
\end{abstract}

Conclusions: Eulerian video magnification provides a technique to extract inapparent temporal signals from videographic material of animals. This can be applied in experimental and comparative physiology where contactbased recordings (e.g., heart rate) cannot be acquired.

Keywords: Videographic material, Signal processing, Heart rate, Respiration rate, Pulse wave velocity, Embryonic development, Infrasound

\section{Background}

The world is teeming with invisible signals and information imperceptible to humans. Some animals have evolved sensory systems that allow them to take advantage of these, seemingly, insensible signals that fall

\footnotetext{
* Correspondence: henrik@clin.au.dk

${ }^{1}$ Nancy E. and Peter C. Meinig School of Biomedical Engineering, Cornell University, 304 Weill Hall, Ithaca, NY 14853-7202, USA

2Department of Clinical Medicine, Aarhus University, Palle Juul-Jensens

Boulevard 99, 8200 Aarhus N, Denmark

Full list of author information is available at the end of the article
}

outside the range of human perception. Examples of this are ultrasound perception in bats (visionless flight first described in [1], and ultrasound production/perception first described in [2, 3]) and cetaceans [4]; electroreception in a range of animals such as several bony fishes [5], elasmobranchs [6], and even mammals [7]; and magnetoreception in a range of migratory birds [8] and other species (reviewed in [9]). Recently, a novel technique named Eulerian video magnification (EVM) was developed to magnify minute color changes or motion,

(c) The Author(s). 2019 Open Access This article is distributed under the terms of the Creative Commons Attribution 4.0 International License (http://creativecommons.org/licenses/by/4.0/), which permits unrestricted use, distribution, and 
invisible to the naked eye, from videographic material [10]. In this technique, a video sequence is spatially decomposed (i.e., separated into different spatial frequency bands) and temporally filtered with an adjustable band-pass filter and the resulting signal is amplified to reveal hidden temporal variations (Fig. 1a. For full mathematical background, see [10]). The result of this procedure is a marked magnification of inherent local fluctuations in the video sequence, which can reveal the subtle skin color changes in the human face related to increased capillary perfusion after left ventricular ejection allowing for an easy heart rate $\left(f_{\mathrm{H}}\right)$ measurement or render visible the faint motions related to breathing in infants [10]. A previous attempt has been made to extract human $f_{\mathrm{H}}$ from video material [11]; however, this procedure relies on sophisticated face-tracking algorithms and is unlikely to be applicable to other species. Likewise, other motion magnification techniques have been developed [12, 13]. However, these follow the Lagrangian perspective (with reference to fluid dynamics where the trajectory of particles is tracked over time, as opposed to the Eulerian perspective where the properties of a fluid evolve over time) and are subject to produce artifacts when applied to the complicated and unpredictable motions of animals. So far, EVM has not been used in any studies in the field of experimental biology, likely because the mathematical fundament of this technique is somewhat complicated and may be perceived by biologists as difficult to apply. However, the advantages of amplifying our sense of vision to perceive minute fluctuations with this tool should outweigh these concerns.

Being able to acquire a reliable and completely noninvasive measurement of $f_{\mathrm{H}}$ from video material may, arguably, be the most valuable application of EVM in experimental biology. Assuming that an animal is in metabolic steady-state in which energy is constantly replenished by aerobic metabolism, the metabolic rate $(M R)$ and the rate of oxygen consumption $\left[\dot{V}\left(\mathrm{O}_{2}\right)\right]$ may be assumed to be equivalent. Fick's convection equation for the cardiovascular system [14]:

$$
\dot{V}\left(\mathrm{O}_{2}\right)=f_{\mathrm{H}} \times V_{\mathrm{S}} \times\left[C_{\mathrm{a}}\left(\mathrm{O}_{2}\right)-C_{\mathrm{v}}\left(\mathrm{O}_{2}\right)\right]
$$

where $V_{\mathrm{S}}$ is the stroke volume, $C_{\mathrm{a}}\left(\mathrm{O}_{2}\right)$ is the $\mathrm{O}_{2}$ content of arterial blood, and $C_{\mathrm{v}}\left(\mathrm{O}_{2}\right)$ is the $\mathrm{O}_{2}$ content of venous blood, summarizes the relationship between $f_{\mathrm{H}}$ and $\dot{V}\left(\mathrm{O}_{2}\right)$, and if the oxygen pulse $\left\{V_{\mathrm{S}} \times\left[C_{\mathrm{a}}\left(\mathrm{O}_{2}\right)-\right.\right.$ $\left.\left.C_{\mathrm{v}}\left(\mathrm{O}_{2}\right)\right]\right\}$ is constant or varies in a predictable way with changes in $f_{\mathrm{H}}$, then measurements of $f_{\mathrm{H}}$ can be used to evaluate $M R$ of animals in both laboratory and field conditions [15]. The $f_{\mathrm{H}^{-}}-M R$ relationship has been investigated in numerous studies on vertebrates of different classes under different circumstances, e.g., resting, walking, swimming, diving, flying, thermoregulating, and digesting (for review see [15]). Generally, a positive correlation between $f_{\mathrm{H}}$ and $M R$ can be found in mammals, birds, and reptiles where $f_{\mathrm{H}}$ is the dominant component of changes in $\dot{V}\left(\mathrm{O}_{2}\right)$ [15]. In fishes (and presumably in amphibians, though this is not wellsupported by literature), the general consensus has for a long time been that $\dot{V}\left(\mathrm{O}_{2}\right)$ is strongly regulated by the oxygen pulse (largely based on a classical study [16]), and therefore, a $f_{\mathrm{H}}-M R$ relationship is more challenging to establish [17]. However, this view has recently been challenged, and it is now recognized that tachycardia at rest is likely to have biased many of the older studies of $f_{\mathrm{H}}$ in fish due to short recovery times after surgery and disturbances $[18,19]$.

Traditionally, externally attached or implantable biologging devices that record electrocardiogram or acoustic information are used to acquire $f_{\mathrm{H}}$ in the laboratory or in the wild. The advantage of this is continuous logging and the ability to monitor the animal in situations where the researcher cannot be present (extreme dives, flying, etc.). The disadvantage is that the device first has to be attached to, or implanted into, the animal which requires some degree of manual handling and often involves anesthesia. This may affect measurements for some time after perturbation and even continuously if the device affects the physiological or behavioral condition of the animal (e.g., [20]). Additionally, in most cases, these devices must be removed to recover the data, requiring a second manipulation or capture of the subject. Therefore, in some situations where filming may be applicable (either active filming or using passive "camera traps"), $f_{\mathrm{H}}$ obtained by EVM may be desirable. The temporal occurrence of color changes related to the propagating pulse wave associated with a heartbeat also carries information about pulse wave velocity. Additionally, other minute color changes or motions related to animal physiology or behavior such as breathing and sound production may be obtained by this technique.

In this study, we describe how EVM can be used to obtain inapparent signals, mostly focusing on $f_{\mathrm{H}}$. This is established through a series of laboratory-based experiments of heart rate detection in neotenic salamanders, fish, embryonic chickens, and mice, and finally with examples of captive (zoo) and free-ranging wildlife situations in which hidden signals can be magnified and detected.

\section{Results}

First, we wanted to test if EVM would allow for $f_{\mathrm{H}}$ measurements under laboratory conditions where camera and light settings were easily adjustable. As the primary animal model for these initial steps, we selected the axolotl [Ambystoma mexicanum (Shaw and Nodder, 1798)], a Mexican caudate amphibian renowned for its tissue 


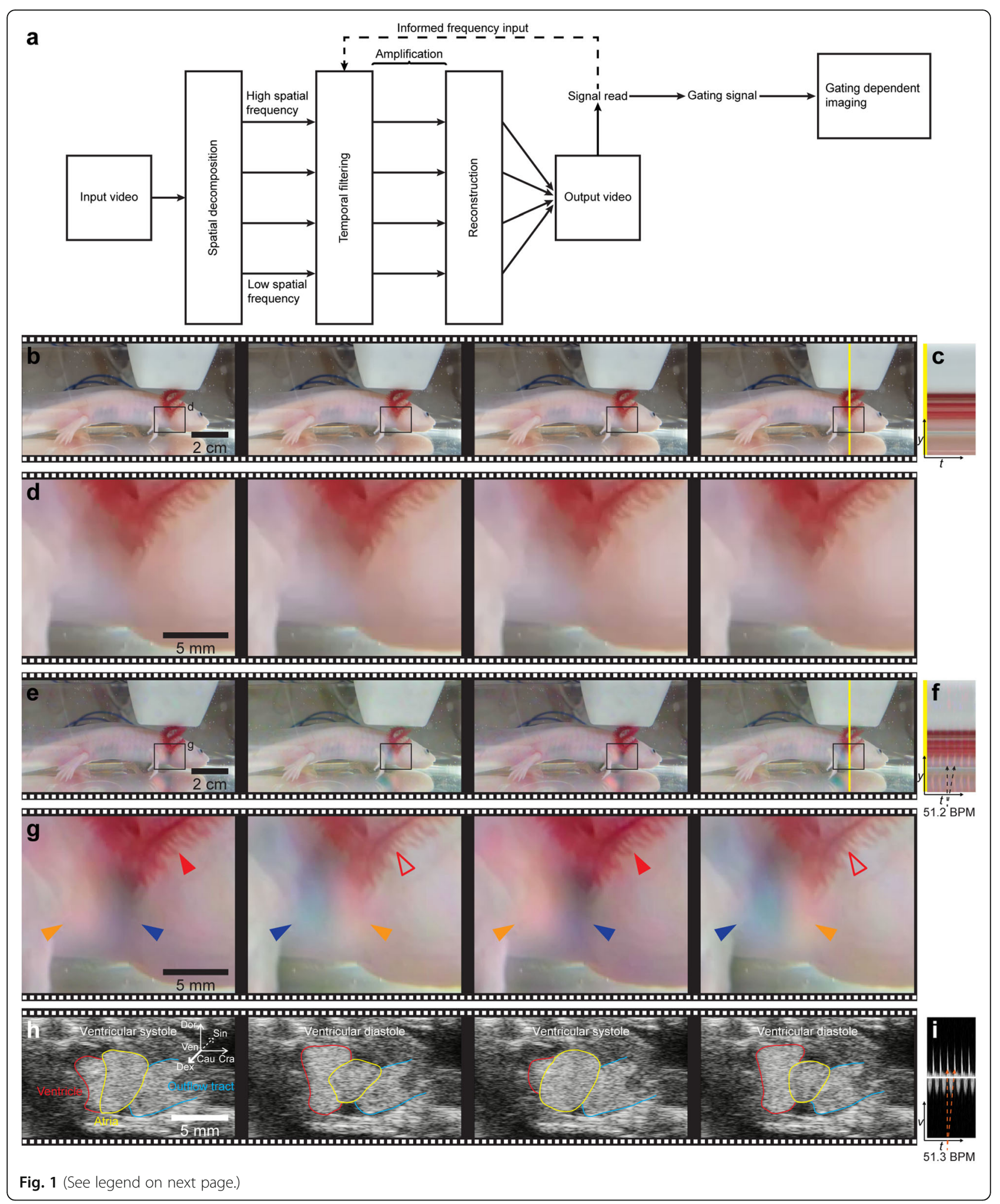


(See figure on previous page.)

Fig. 1 Overview and example of Eulerian video magnification procedure. a Block diagram representing the processing steps of a Eulerian Video Magnification procedure resulting in a signal read of the signal of interest or a gating signal for another imaging procedure. $\mathbf{b}$ Four frames from original input video source of anesthetized axolotl (Additional file 1, left trace). c Vertical scan line from the input source over time. $\mathbf{d}$ Magnification of box in $\mathbf{b}$ showing the cardiac region of the input video. No obvious color changes resulting from the beating heart. e The same four frames as in $\mathbf{b}$ with the axolotl's pulse signal magnified. $\mathbf{f}$ Same scan line over time as in $\mathbf{c}$ but on color-magnified video. Color fluctuations are visible. $\mathbf{g}$ Magnification of box in $\mathbf{e}$. Color changes are present over time in the cardiac region. $\mathbf{h}$ Four frames of brightness mode echocardiography recorded simultaneously with input video. The frames match the same time points as in $\mathbf{b}, \mathbf{d}, \mathbf{e}, \mathbf{g}$. i Pulsed-wave Doppler recording of blood flow in the ventricle. Note that peak flow matches lines in $\mathbf{f}$, demonstrating that color changes in magnified video result from the beating heart

regenerative potential [21-23]. The axolotl is easily amenable to immersion-based anesthetics such as benzocaine (ethyl 4-aminobenzoate), MS-222 (ethyl 3aminobenzoate methanesulfonic acid), and propofol (2, 6-diisopropylphenol) [24] and does not require constant gill irrigation which reduces motion noise during video recording. The axolotl exists in different color strains, e.g., dark green/brown wild type, white leucistic, and albino, allowing for evaluation of the methodology on different color types. Additionally, the axolotl not only exhibits skin covered deep vascular beds but also very superficial vascular networks in their external gills which should result in areas with a high degree of color change during blood movement. To demonstrate the EVM method, we anesthetized a leucistic axolotl (body mass (BM) $10.73 \mathrm{~g}$, total length (TL) $11.8 \mathrm{~cm}$ ) in $200 \mathrm{mg} / \mathrm{l}$ benzocaine and video recorded (see Table 1 for video specifications) the water-covered and sedated animal from a lateral viewpoint, while simultaneously acquiring an echocardiographic signal for validation of $f_{\mathrm{H}}$ (Fig. 1). Although the original video recording did not display any obvious color changes or motions related to the animal's heartbeat (Fig. 1b-d; Additional file 1, left trace), EVM-based color magnification (Fig. 1e-g; Additional file 1 , right trace, first part) and motion magnification (Additional file 1, right trace, second part) both revealed rhythmic color changes and motions on the skin surface directly adjacent to the position of the heart and on the external gills, changing with the same frequency as the heartbeat signal acquired with ultrasonographic imaging (Fig. 1h-i; Additional file 1, third part and sound trace for all three parts).

\section{Optimization of EVM and physiological measurements}

Next, we wanted to test if heartbeat signal detection could be optimized by applying a two-stage frequencyinformed EVM procedure [Fig. 1a, i.e., first allow for a wide passband to pick up a heartbeat signal with an unknown frequency and then reprocess the video with a much narrower band-pass filter centered on the measured $f_{\mathrm{H}}$ to optimize signal to noise ratio (SNR)], and by selecting a specific channel (red, green, or blue) of the magnified video with standard 8-bit RGB colors. Additionally, we wanted to demonstrate a simple cardiovascular physiology relevant setup in which EVM-acquired $f_{\mathrm{H}}$ could be obtained for resting, exercised, and anesthetized axolotls. We studied six leucistic axolotls $(\mathrm{BM} 9.59 \pm 1.68 \mathrm{~g}$, TL $11.52 \pm 0.63 \mathrm{~cm})$ that were video recorded from a dorsal viewpoint after $48 \mathrm{~h}$ of fasting and $24 \mathrm{~h}$ of undisturbed rest (see Table 1 for video specifications), then gently subjected to echocardiographic $f_{\mathrm{H}}$ validation by submerging a remotely operated ultrasound transducer to a level $2 \mathrm{~cm}$ above the thoracic area of the animal. Thereafter the axolotls were exercised in a swimming respirometer to maximal swimming capacity by gradually increasing the water current until the moment when the animal was not able to produce sufficient force to maintain position against the water current. Video recordings were made directly after exercise. Echocardiographic $f_{\mathrm{H}}$ validation was not possible at this stage as the exercised axolotls were so agitated that positioning of the transducer in the required distance $(<2 \mathrm{~cm})$ from the thoracic area resulted in escape behavior. Following an additional 24 $\mathrm{h}$ of undisturbed rest, benzocaine was added to the aquaria via catheters to induce anesthesia. After full anesthesia was reached (60 min [24]), video recordings were made of the sedated axolotls and these were subsequently subjected to echocardiographic $f_{\mathrm{H}}$ validation as described above. Again, EVM revealed a rhythmic color change signal in the gill region of all animals (exemplified in Fig. 2a-c). Applying a two-stage frequency-informed EVM procedure generated magnified videos with less noise (Fig. 2d, e, compare Fig. 2b with Fig. 2e; Additional file 2). Channel splitting revealed marked differences in signal amplitude in the red, green, and blue channels (Fig. 2f). Under the specific experimental light setting and the color pattern of the leucistic axolotls, the green channel displayed the largest signal amplitude (exemplified in Fig. $2 \mathrm{f}-\mathrm{j}$ ). In all subsequent experiments, the two-stage frequencyinformed EVM and the channel selection procedure was incorporated to optimize band-pass filter and select optimal channel mode (red, green, blue, or all channels combined).

Benzocaine has a well-known tachycardiac effect in axolotls [24]; therefore, it was not surprising that both exercise and benzocaine-induced anesthesia resulted in 
Table 1 Specifications for included videos and Eulerian video magnification parameters

\begin{tabular}{|c|c|c|c|c|c|c|c|c|}
\hline $\begin{array}{l}\text { Related } \\
\text { figure of } \\
\text { video } \\
\text { material }\end{array}$ & $\begin{array}{l}\text { Number } \\
\text { of videos }\end{array}$ & $\begin{array}{l}\text { Spatial resolution } \\
\left(p x^{2}\right)\end{array}$ & $\begin{array}{l}\text { Temporal } \\
\text { resolution } \\
\text { (FPS) }\end{array}$ & $\begin{array}{l}\text { Magnification } \\
\text { type (C or M) }\end{array}$ & $\begin{array}{l}\text { Initial EVM band- } \\
\text { pass filter ( } \mathrm{Hz})\end{array}$ & $\begin{array}{l}\text { Informed EVM } \\
\text { band-pass } \\
\text { filter (Hz) }\end{array}$ & $\begin{array}{l}\text { Channel } \\
\text { (Com., } \\
\text { R, G, B) }\end{array}$ & $\begin{array}{l}\text { Magnification } \\
\text { level }\end{array}$ \\
\hline $\begin{array}{l}\text { Figure 1b- } \\
\mathrm{g}\end{array}$ & 1 & $1920 \times 1080$ & 30 & $\mathrm{C}$ and $\mathrm{M}$ & $0.1-3.0$ & $0.8-0.9$ & $\begin{array}{l}\text { All, } \\
\text { Com. }\end{array}$ & 100 \\
\hline Figure 2a- & 1 & $1920 \times 1080$ & 30 & $C$ & $0.5-1.2$ & $0.6-0.8$ & $\begin{array}{l}\text { All, } \\
\text { Com., G }\end{array}$ & 100 \\
\hline $\begin{array}{l}\text { Figure 2k- } \\
x\end{array}$ & 18 & $1920 \times 1080$ & 30 & $C$ & $0.2-1.2$ & $\begin{array}{l}0.2-0.5 \text { (rest), } 0.6-0.8 \\
\text { (exercise and anesthesia) }\end{array}$ & All, G & 100 \\
\hline Figure 3 & 6 & $\begin{array}{l}3840 \times 2160 \\
2560 \times 1440 \\
1920 \times 1080 \\
1920 \times 1080 \\
1280 \times 720 \\
640 \times 480\end{array}$ & $\begin{array}{l}30,30,60,30 \\
30,30\end{array}$ & C & $0.5-1.2$ & $0.6-0.8$ & All, G & 100 \\
\hline $\begin{array}{l}\text { Figure } 4 a- \\
c\end{array}$ & 1 & $1920 \times 1080$ & 30 & M & $0.2-0.8$ & $0.3-0.5$ & All, G & 10,000 \\
\hline $\begin{array}{l}\text { Figure } 4 d- \\
k\end{array}$ & 1 & $1920 \times 1080$ & 30 & $C$ & $0.2-1.2$ & $0.3-0.5$ & All, G & 100 \\
\hline Figure 5 & 4 & $1920 \times 1080$ & 30,60 & $C$ & $1.8-3.0$ & $1.8-2.4$ & All, B & 50 \\
\hline $\begin{array}{l}\text { Figure 6a, } \\
\mathrm{b}\end{array}$ & 1 & $1280 \times 720$ & 120 & $C$ & $0.5-1.2$ & $0.8-0.9$ & $\begin{array}{l}\text { All, } \\
\text { Com. }\end{array}$ & 100 \\
\hline $\begin{array}{l}\text { Figure 6d- } \\
\text { h }\end{array}$ & 1 & $1280 \times 720$ & 120 & $C$ & $0.9-1.3$ & $1.0-1.1$ & $\begin{array}{l}\text { All, } \\
\text { Com. }\end{array}$ & 100 \\
\hline $\begin{array}{l}\text { Figure 6i- } \\
\text { m }\end{array}$ & 1 & $1280 \times 720$ & 120 & $C$ & $0.9-1.3$ & $1.0-1.1$ & $\begin{array}{l}\text { All, } \\
\text { Com. }\end{array}$ & 100 \\
\hline $\begin{array}{l}\text { Figure 7a- } \\
\mathrm{c}\end{array}$ & 1 & $1920 \times 1080$ & 30 & M & $1.8-2.5$ & $1.9-2.3$ & All, B & 100 \\
\hline $\begin{array}{l}\text { Figure } 7 d- \\
g\end{array}$ & 1 & $1280 \times 720$ & 30 & $C$ & $0.5-0.8$ & $0.7-0.8$ & All, G & 100 \\
\hline $\begin{array}{l}\text { Figure 8a- } \\
\mathrm{n}\end{array}$ & 156 & $1280 \times 720$ & 120 & $C$ & $\begin{array}{l}1.3-2.9 \text { (day } 1-3), 1.5-3.2 \\
\text { (day } 3.5-5.0), \\
2.7-4.8 \text { (day } 6.0-9.0 \text { ) }\end{array}$ & $\begin{array}{l}1.5-2.8 \text { (day } 1-3 \text { ), } \\
1.5-3.2 \text { (day 3.5-5.0), } \\
3.2-4.8 \text { (day 6.0-9.0) }\end{array}$ & $\begin{array}{l}\text { All, } \\
\text { Com. }\end{array}$ & 100 \\
\hline $\begin{array}{l}\text { Figure } 80- \\
\vee\end{array}$ & 1 & $1920 \times 1080$ & 30 & $C$ & $1.0-2.2$ & $1.5-1.9$ & $\begin{array}{l}\text { All, } \\
\text { Com. }\end{array}$ & 100 \\
\hline Figure 10 & 4 & $1920 \times 1080$ & 50,60 & M & $0-30$ & $0-30$ & $\begin{array}{l}\text { All, } \\
\text { Com. }\end{array}$ & 300 \\
\hline Figure 11 & 1 & $1920 \times 1080$ & 50 & M & $0-25$ & $0-25$ & $\begin{array}{l}\text { All, } \\
\text { Com. }\end{array}$ & 500 \\
\hline
\end{tabular}

"Related figure of video material" specifies what figure parts the video has been used for. "Channel" specifies which channel of magnified videos that were analyzed for the initial magnification (for all video material, this was done for all channels including the combined channel signal) and for the subsequent informed processing

px pixel, FPS frames per second, Com. combined, $R$ red, $G$ green, $B$ blue

increases in $f_{\mathrm{H}}$ (Fig. 2k-x; Additional file 3) and gill filament dilation (see inserts in Fig. 2k, n, q). A one-sample $t$ test showed that the difference between $f_{\mathrm{H}}$ acquired by EVM and echocardiography in both resting and anesthetized conditions was not statistically significantly different from $0(p=0.30)$, thereby validating the accuracy of the EVM method. One-way ANOVA showed that EVMacquired $f_{\mathrm{H}}$ differed statistically significantly between conditions (rest, exercise, and anesthesia) $[\mathrm{F}(2,15)=$ 41.88, $p<0.01]$. A Tukey Honestly Significant Difference post hoc test revealed that resting $f_{\mathrm{H}}$ was statistically lower than $f_{\mathrm{H}}$ following exercise and during anesthesia
[Fig. 2x. Mean $f_{\mathrm{H}}$ (rest, EVM) $\pm 95 \%$ confidence interval $(\mathrm{CI})=22.23 \pm 5.18$ beats per minute $(\mathrm{BPM})$, mean $f_{\mathrm{H}}$ (exercise, $\mathrm{EVM}) \pm 95 \% \mathrm{CI}=39.44 \pm 2.38 \mathrm{BPM}$, mean $f_{\mathrm{H}}$ (anesthesia, $\mathrm{EVM}) \pm 95 \% \mathrm{CI}=42.99 \pm 1.19 \mathrm{BPM} . f_{\mathrm{H}}$ (rest, EVM) vs. $f_{\mathrm{H}}$ (exercise, EVM): $p<0.001, f_{\mathrm{H}}$ (rest, EVM) vs. $f_{\mathrm{H}}$ (anesthesia, EVM): $p<0.001$ ], but $f_{\mathrm{H}}$ levels at exercise and anesthesia were not statistically significantly different from each other $\left[f_{\mathrm{H}}\right.$ (exercise, EVM) vs. $f_{\mathrm{H}}$ (anesthesia, EVM): $p=0.33$ ].

Next, we wanted to test the importance of spatial and temporal resolution of video material intended for the EVM procedure since this has major implication on 


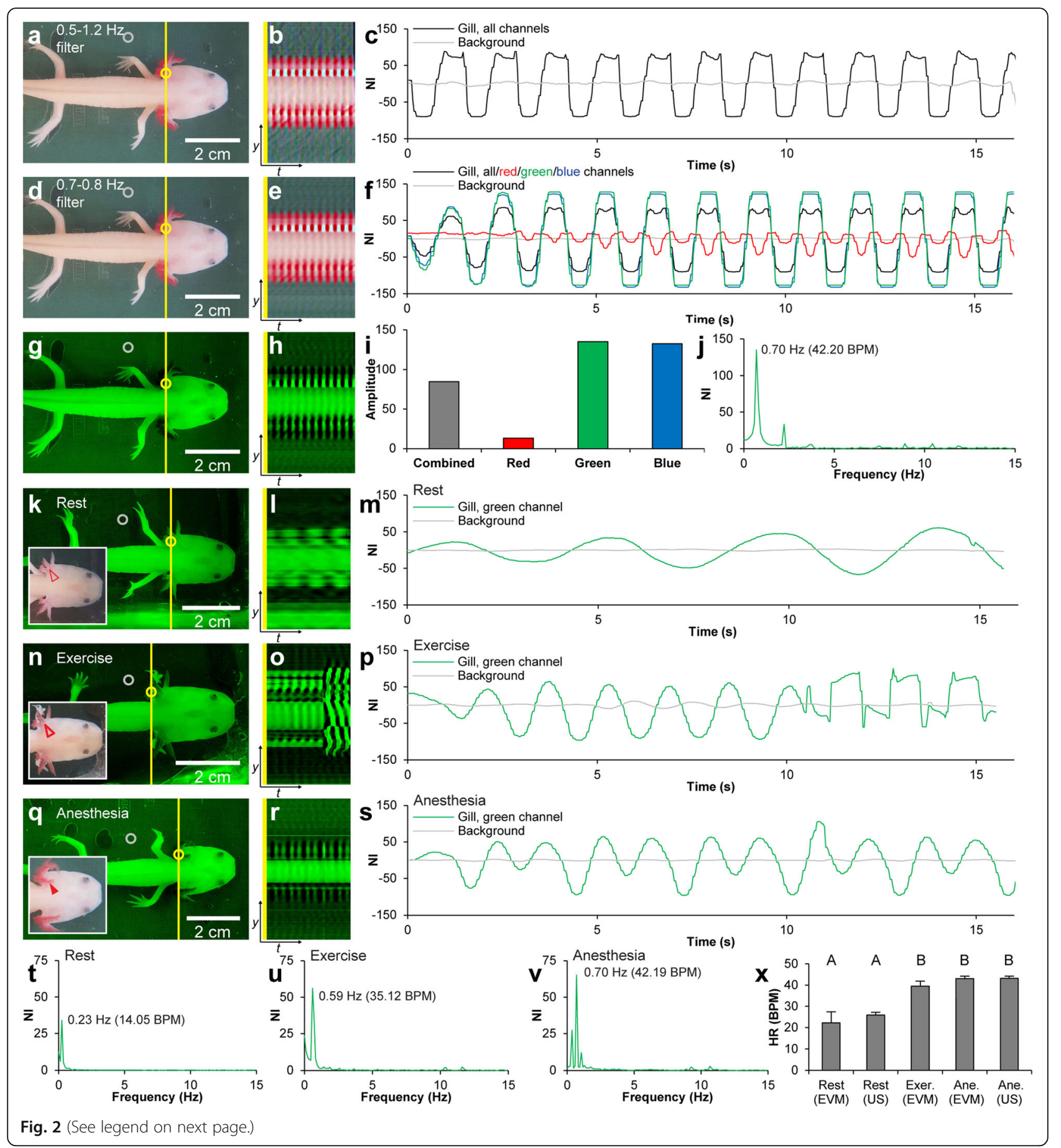




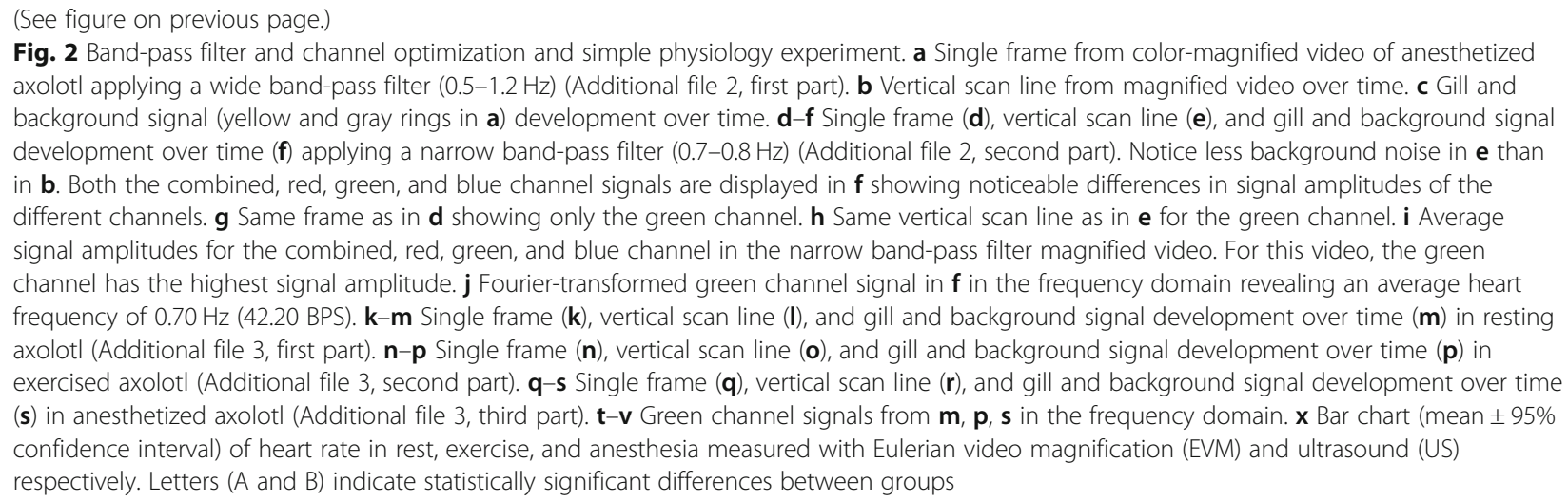

video file size and manageability. We recorded the same scene, an anesthetized leucistic axolotl positioned in a supine position exposing the thoracic region and the underlying beating heart, using six standard video frame formats: ultra-high definition $\left[3840 \times 2160\right.$ pixels $(\mathrm{px})^{2}$, 30 frames per second (FPS)], quad-high definition $\left(2560 \times 1440 \mathrm{px}^{2}, 30 \mathrm{FPS}\right)$, full-high definition with high speed $\left(1920 \times 1080 \mathrm{px}^{2}, 60 \mathrm{FPS}\right)$, full-high definition $\left(1920 \times 1080 \mathrm{px}^{2}, 30 \mathrm{FPS}\right)$, high definition $(1280 \times 720$ $\left.\mathrm{px}^{2}, 30 \mathrm{FPS}\right)$, and video graphics array $\left(640 \times 480 \mathrm{px}^{2}, 30\right.$ FPS). Subsequent to EVM, all video formats allowed for a clear heartbeat signal detection and $f_{\mathrm{H}}$ measurement (Fig. 3; Additional file 4).

\section{Eulerian video magnification under non-optimal conditions}

In colored axolotls, subtle color changes in the thoracic region are not as pronounced as in leucistic and more translucent individuals, which likely applies to other species as well. However, our initial test on the axolotl revealed that even small rhythmic motions in the front limbs related to blood movement in the brachial arteries could be detected with EVM-based motion magnification (Additional file 1). Therefore, we wanted to test the applicability of EVM-based motion magnification to detect $f_{\mathrm{H}}$ via movements of the front limb in a dark brown wild-type axolotl. A large wild-type axolotl (BM $61.90 \mathrm{~g}$, TL $19.7 \mathrm{~cm}$ ) was anesthetized in $200 \mathrm{mg} / \mathrm{l}$ benzocaine, video recorded (for video specifications see Table 1), and the video was subsequently processed for motion magnification. By placing the image probe for motion detection directly on the interface of the upper limb and background in the magnified video, heart rate detection was possible (Fig. 4a-c; Additional file 5).

To mimic an experimental setup in which $f_{\mathrm{H}}$ needs to be monitored in multiple animals at the same time with a limited number of cameras and with non-optimal light and background settings, we used the same six leucistic animals as in the rest/exercise/anesthesia experiment.
The six axolotls were housed in individual plastic aquaria and placed on a gray linoleum background. Following $48 \mathrm{~h}$ of fasting and $24 \mathrm{~h}$ of undisturbed rest, a video recording containing all six animals was performed (see Table 1 for video specifications). Although axolotls are relatively sedentary animals, the chances of encountering movements during recording increases with the number of animals being recorded at the same time and the duration of recording. Figure $4 \mathrm{~d}$ and Additional file 6 display a recording in which five out of six animals did not move during the 15-s recording, whereas animal 6 did move for the last $5 \mathrm{~s}$. In spite of little contrast between axolotls and background, and pronounced reflections and shadows in the scene (Fig. 4d), it was still possible to acquire $f_{\mathrm{H}}$ for all six animals [mean $f_{\mathrm{H}}$ (rest, $\mathrm{EVM}) \pm 95 \% \mathrm{CI}=22.27 \pm 4.24 \mathrm{BPM} ;$ Fig. $4 \mathrm{~d}-\mathrm{k}]$ and a paired $t$ test showed that resting heart rate was not significantly different from previous individual recordings of the same animals $(p=0.99)$.

In order to test if EVM allowed for $f_{\mathrm{H}}$ detection in a more active ectotherm than the axolotl, we used conscious, unrestrained zebrafish [Danio rerio (Hamilton, 1822)]. Forty-five adult ( $>4$ months of age) mixed sex, wild-type zebrafish (representative group of 13 animals euthanized for parallel research had BM $457.15 \pm$ $109.09 \mathrm{mg}$, TL $39.08 \pm 2.02 \mathrm{~mm}$ ) were housed in a $200-1$ aquarium with fully oxygenated water $(90 \mathrm{l})$ at $27.6{ }^{\circ} \mathrm{C}$. Following $36 \mathrm{~h}$ of fasting and $24 \mathrm{~h}$ of undisturbed rest to minimize post absorptive heart rate changes and disturbance, video recordings were performed with a remotely controlled camera (see Table 1 for video specifications). Although zebrafish are active animals, it was possible to acquire four videos of eight separate instances over a 15 -min period in which the movements of the fish, although rapid (mean movement speed \pm $95 \% \mathrm{CI}=9.36 \pm 2.44 \mathrm{TL} / \mathrm{s}$ ), were brief enough and out of the frequency range of expected $f_{\mathrm{H}}$ that EVM allowed for $f_{\mathrm{H}}$ extraction (Fig. 5; Additional file 7; mean $\left.f_{\mathrm{H}}(\mathrm{EVM}) \pm 95 \% \mathrm{CI}=127.57 \pm 7.87 \mathrm{BPM}\right)$. 

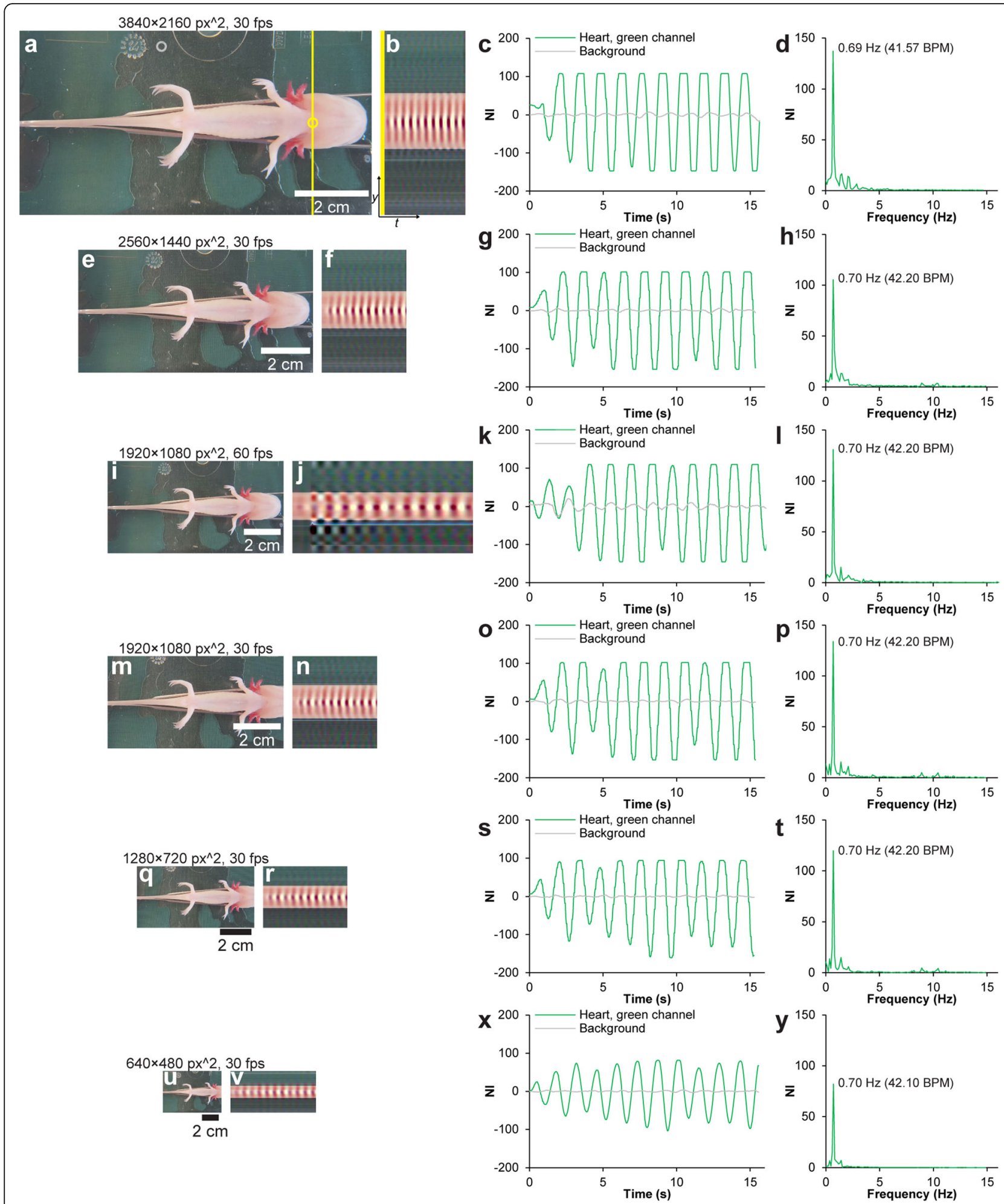

Fig. 3 (See legend on next page.) 


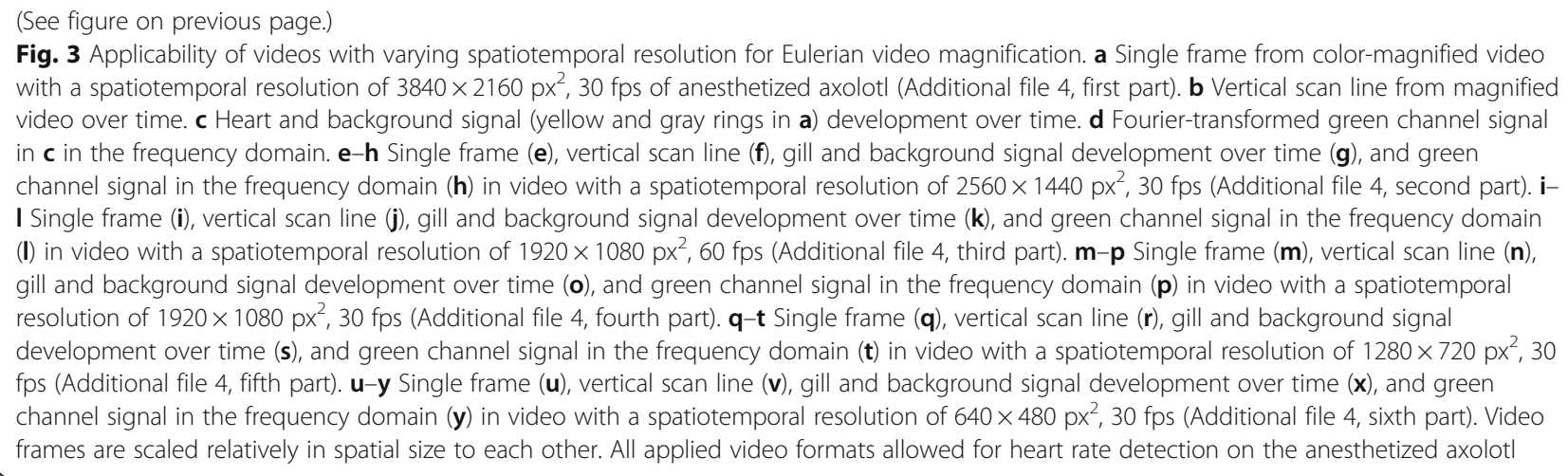

\section{Pulse wave velocity measurement with EVM}

Eulerian video magnification operates on a pixel basis; therefore, a time shift between two or more rhythmic temporal phenomena, such as a propagating pulse wave causing a directional color change, can in theory be detected at different locations given that the temporal resolution of the camera is sufficient to detect the time gap between the pulse arriving at the locations of interest. To test if EVM allowed for detection of pulse wave velocity (PWV), we first examined the low pressure, low PWV vascular system of the amphibian axolotl. An anesthetized leucistic axolotl (BM $11.30 \mathrm{~g}$, TL $11.7 \mathrm{~cm}$ ) was positioned on the side (Fig. 6a) and recorded at a high frame rate (120 FPS, see Table 1 for additional specifications). The recording was processed with EVM (Additional file 8), and color fluctuations were measured at two positions in the vascular system (Fig. 6b, c). The amphibian dorsal aorta (DA) originates from the efferent gill arteries and runs in the caudal direction along the long axis of the animal (Fig. 6d). A proximal DA and a distal DA position separated by $64.22 \mathrm{~mm}$ was selected, and the time gap between signal peaks and the pulse transit time (PTT) was measured as mean PTT $=71.22$ ms resulting in a mean $\mathrm{PWV}=0.90 \mathrm{~m} / \mathrm{s}$. To test the procedure on a high pressure, high PWV vascular system, we examined a resting male human being (Homo sapiens Linnaeus, 1758) [BM $95 \mathrm{~kg}$, TL $184 \mathrm{~cm}, f_{\mathrm{H}}$ (rest, EVM) = 63.33 $\mathrm{BPM}$ ] and repeated the procedure described above on the throat (PWV in the left arteria carotis communis) (Fig. 6d-i; Additional file 9, first part), and the upper arm (PWV in the right arteria brachialis) (Fig. 6i-n; Additional file 9, second part). Time shift at the two most distant positions of the arteria carotis communis with detectable color fluctuations (separated by 96.13 $\mathrm{mm}$ ) was close to the temporal detection limit (Fig. 6g, h) and resulted in a mean $\mathrm{PTT}=21.95 \mathrm{~ms}$ and mean $\mathrm{PWV}=4.38 \mathrm{~m} / \mathrm{s}$. The two upper arm locations (armpit and elbow separated by $264.08 \mathrm{~mm}$ ) displayed an easily detectable phase shift (Fig. 61, m) with a mean PTT = $72.86 \mathrm{~ms}$ and mean PWV $=3.62 \mathrm{~m} / \mathrm{s}$.

\section{Eulerian video magnification of non-dedicated videographic material}

To test the applicability of EVM on animal videographic material that was not produced with the purpose of EVM, two online videos were located and analyzed with permission from the producers. The first video displayed a basking sand lizard (Lacerta agilis Linnaeus, 1758) (Fig. 7a). Following motion-enhanced EVM, mean $f_{\mathrm{H}}$ (136.97 BPM) and mean respiration rate (94.83 respirations per minute) could be measured over multiple cycles at the level of the heart and the lungs respectively (Fig. 7b, c). The second video displayed a drinking giraffe [Giraffa camelopardalis (Linnaeus, 1758)] that eventually lifted its head up (Fig. 7d-e). Here, color-enhanced EVM was used to the posterior part of the hind limb where the arteria tibialis posterior runs close to the surface and the fur layer is relatively thin. This allowed for $f_{\mathrm{H}}$ signal detection over five cardiac cycles while the head was down (mean $f_{\mathrm{H}}=42.19$ BPM) (Fig. 7f) and one cardiac cycle when the head was elevated $\left(f_{\mathrm{H}}=45.09\right.$ BPM) (Fig. 7g).

\section{Embryonic heart rate detection and optical gating}

Development of embryonic domestic chickens [Gallus gallus (Linnaeus, 1758)] can be directly observed both by incubation in ovo with a window in the eggshell or in ex ovo cultures in which the eggshell is completely removed $[25,26]$. The developing heart is visible at early stages of development and starts to contract after 29-45 $\mathrm{h}$ of development [Hamburger Hamilton stage $(\mathrm{HH}) 9$ 10] [27-30], allowing for an easy validative measurement of $f_{\mathrm{H}}$ under these incubation conditions. We wanted to test if EVM would allow for $f_{\mathrm{H}}$ detection through the intact eggshell during the first 9 days of embryonic development. A group of ten intact eggs and three windowed eggs (windowed at embryonic day 1.25) were video recorded (for video specifications see Table 1) at 12 time points during the first 9 days of embryonic development [embryonic day 1.25 ( $\mathrm{HH}$ 6-7), $1.5(\mathrm{HH} 10-11), 2.0$ (HH 12-13), 2.38 (HH 17-18), 3.0 (HH 19-20), 3.5 (HH 

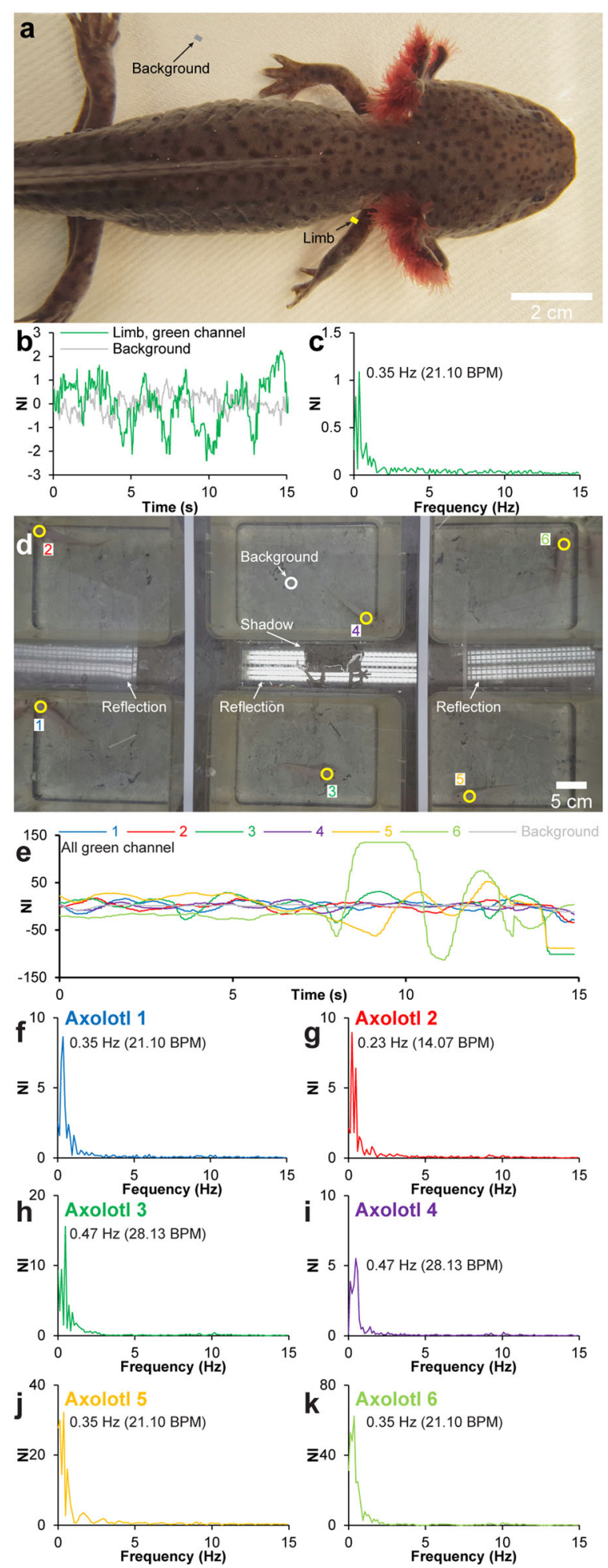

Fig. 4 (See legend on next page.) 
(See figure on previous page.)

Fig. 4 Heart rate detection in colored animal and in multi-animal setup. a Single frame from motion magnified video of brown wild-type axolotl (Additional file 5). b Proximal limb and background signal (yellow and gray lines in a) development over time (green channel). c Fouriertransformed green channel signal in $\mathbf{b}$ in the frequency domain. Although the enhanced motion signal related to the heart beat of the colored animal is less pronounced relatively to the color change signal in leucistic animals (see Figs. 1, 2 and 3), a heart rate measurement is still measureable. $\mathbf{d}$ Single frame from color-magnified video of a multi-animal setup containing six resting axolotls (Additional file 6). e Cervical and background signal (yellow and white rings in $\mathbf{d}$ ) development over time of each of the six axolotls in $\mathbf{d}$ (green channel). $\mathbf{f}-\mathbf{k}$ Fourier-transformed green channel signals in $\mathbf{e}$ in the frequency domain. Resting heart rate varied between 14.07 and 28.13 BPM

21-23), 4.0 (HH 24), 5.0 (HH 27), 6.0 ( $\mathrm{HH} 29), 7.0(\mathrm{HH}$ 31), 8.0 ( $\mathrm{HH} \mathrm{34),} 9.0$ ( $\mathrm{HH} 35)$. For intact eggs, the $\mathrm{HH}$ stage could only be assumed]. Video recordings were subsequently processed with EVM to enhance color changes associated with the heartbeat at the position of the heart and at the vitelline vessels (Fig. 8a-f, Additional file 10). One-way ANOVA showed that both heart and vitelline vessel SNR were statistically significantly different for different embryonic time points for both intact and windowed eggs [intact egg, heart: $F$ (11, $108)=21.40, p<0.01$; intact egg, vessels: $F(11,108)=$ $3.95, p<0.01$; windowed egg, heart: $F(11,24)=3.01, p=$ 0.01 ; windowed egg, vessels: $F(11,24)=8.27, p<0.01$ ] Post hoc tests using the Bonferroni correction $(\alpha=0.05 /$ $12=0.0042)$ showed that SNR was statistically significantly larger than 1 for the heart measurements on intact eggs at embryonic day $2.38(p=0.0014), 3.0(p<$ $0.0008), 3.5(\mathrm{p}<0.0008)$, and $4.0(p=0.0013)$ (Fig. 8g) and for the vitelline vessel measurements on intact eggs at embryonic day $3.0(p<0.0008), 3.5(p=0.0020), 4.0$ $(p=0.0026)$, and $8.0(p=0.0038)$ (Fig. 8g, h). Signal to noise ratio for heart and vessel signals displayed a high degree of variability (Fig. 8g, large 95\% CI error bars), which, based on our observations during the experiment, were likely caused by individual orientation of the embryo relative to the eggshell and variance in eggshell thickness, causing some embryos to produce very low SNR for heart and vitelline vessel signal. For all time points from embryonic day 5.0-9.0, there was at least one embryo in the intact eggshell group that displayed an EVM enhanced heart or vitelline vessel signal with a SNR > 6 (for the windowed egg group at least one embryo per time point in the same time span displayed a heart or vitelline vessel signal with a SNR > 17). Mean $f_{\mathrm{H}}$ was calculated for all time points (Fig. 8i) and a two-way mixed ANOVA showed that there was no statistically significant difference in $f_{\mathrm{H}}$ between the intact eggshell and windowed groups $[F(1,4)=0.84, p=0.41]$, but there was a statistically significant different in $f_{\mathrm{H}}$ between different embryonic time points $[F(8,32)=$ $152.56, p<0.01]$. Six of the selected time points matched embryonic days for which $f_{\mathrm{H}}$ has previously been reported (Lindsey et al., 2014). Bonferroni-corrected $(\alpha=0.05 / 6=0.0083) t$ tests showed that $f_{\mathrm{H}}$ for embryos in intact eggs were statistically significantly different from reference values at embryonic day $3.5(p<0.002)$, $4.0(p<0.002)$, and $9.0(p<0.002)$.

With the knowledge of some embryos/eggs being predisposed as good candidates for EVM, we went on to test if real-time EVM could be used to enhance the heart beat signal through the eggshell and allow for optical gating of an in vivo micro-CT system to acquire volumetric data of the beating heart in selected cardiac phases. Eggs were carefully windowed at embryonic day 3.5 , and the excised cap was maintained for later repositioning. Three embryos with an appropriate orientation that qualified them as good EVM candidates were microinjected in a vitelline vein with $100 \mu \mathrm{l}$ of an in vivo vascular contrast agent (Exitron nano 12000, Miltenyi Biotec, Bergisch Gladbach, Germany) on embryonic day 7.0 (see Fig. 8j for embryo size). The excised eggshell cap was carefully repositioned, and the air phase between egg yolk surface with the floating embryo and the eggshell cap was replaced with Tris-buffered isotonic saline to bring the embryo in close proximity to the eggshell, as in the natural non-windowed condition. The egg was positioned on the in vivo micro-CT scanner bed, candled and heated with a flashlight, and recorded with an endoscope camera. Real-time EVM was used to acquire the color-enhanced heart beat signal, and using a custom made software, a gating signal was fed to the micro-CT system to perform a prospectively gated acquisition of projections with the heart in the same cardiac phase. Two acquisitions were made to capture volumetric data of the heart in the end-diastole and the end-systole phases (Fig. 8k-n, Additional file 11). Stereological examination of left and right ventricle blood volume in end-diastole and end-systole yielded a mean stroke volume (SV) of SV (left ventricle) $\pm 95 \% \mathrm{CI}=$ $1.57 \pm 0.28 \mathrm{~mm}^{3}$ and $\mathrm{SV}$ (right ventricle) $\pm 95 \% \mathrm{CI}=$ $2.40 \pm 1.34 \mathrm{~mm}^{3}$ and mean ejection fractions of EF (left ventricle) $\pm 95 \% \mathrm{CI}=47.17 \pm 6.08 \%$ and $\mathrm{EF}$ (right ventricle) $\pm 95 \% \mathrm{CI}=63.35 \pm 11.62 \%$.

In contrast to the embryonic bird, the embryonic mammal is more difficult to access and observe. To test if a heartbeat signal could be acquired from an embryonic mammal in utero, we examined a pregnant C57BL/6 mouse with 10 embryos (number of embryos validated by post-mortem micro-CT imaging and dissection) at embryonic day 17.5 (E17.5). After 

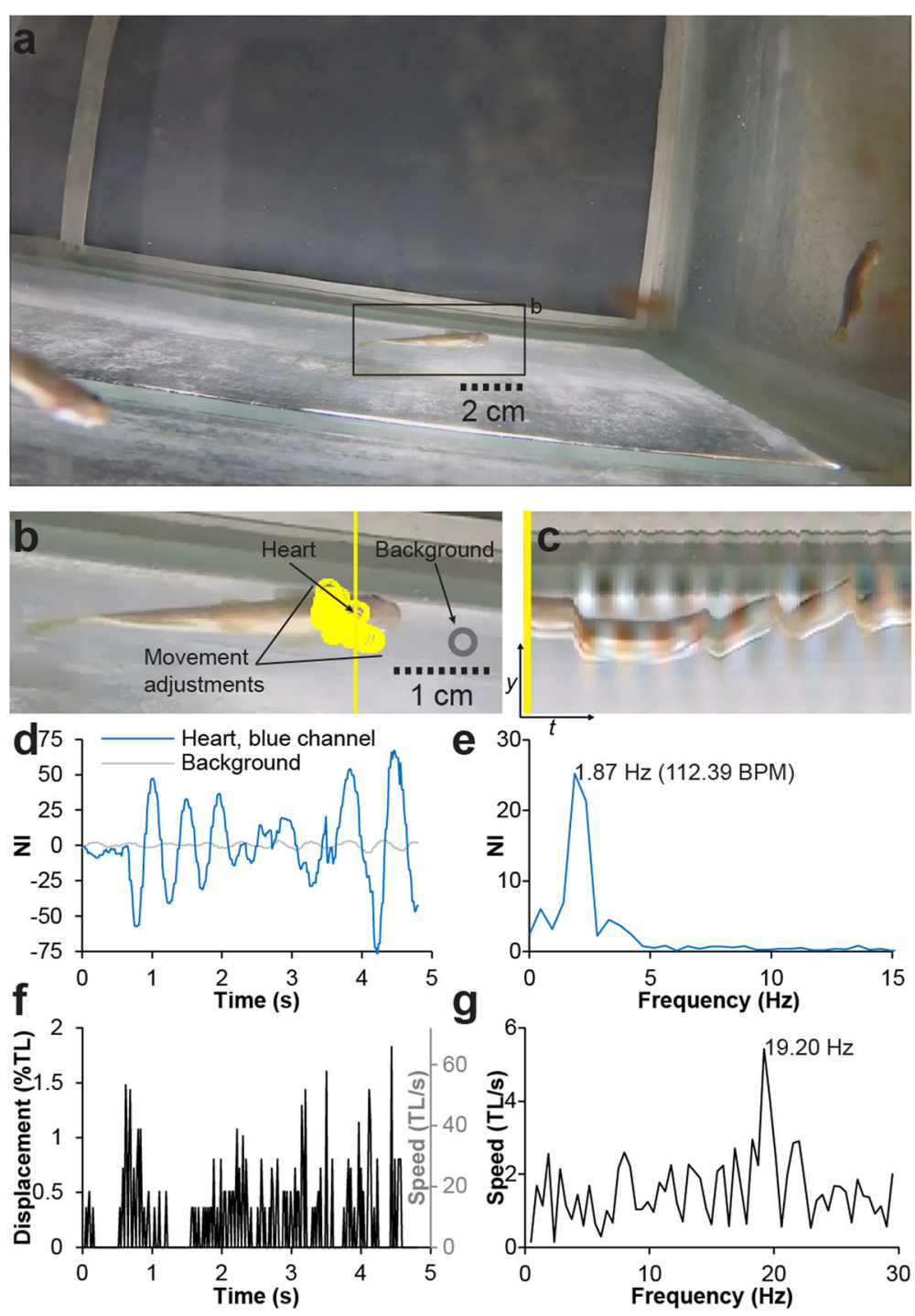

Fig. 5 Heart rate detection in conscious, unrestrained, group-housed zebrafish. a Single frame from color-magnified video of wild-type conscious and unrestrained adult zebrafish (Additional file 7). b Magnification of box in a with a display of regions of interest (yellow rings) tracking the ventral surface of the thoracic region as the fish moves gently. $\mathbf{c}$ Vertical scan line from magnified video over time showing temporal variation of color at the level of the thoracic region. $\mathbf{d}$ Thoracic and background signal (yellow and gray rings in $\mathbf{b}$ ) development over time (blue channel). e Fourier-transformed blue channel signal in $\mathbf{d}$ in the frequency domain. $\mathbf{f}$ Displacement in percent of fish total length (TL) (left vertical axis) and speed in TL/s (right vertical axis) of thoracic region of interest (yellow rings in $\mathbf{b}$ ) during the recording. Although the fish movements are rapid and occur at a high rate, the displacement is small and the rate is out of the frequency range of the beating heart (compare $\mathbf{d}$ with $\mathbf{f}$ ). $\mathbf{g}$ Fouriertransformed speed of displacement signal in $\mathbf{f}$ in the frequency domain. Scale bars in $\mathbf{a}$ and $\mathbf{b}$ are approximations (indicated by dotted lines) based on average zebrafish sizes in the batch since the fish was at an unknown depth in the water column during the recording

induction of anesthesia, the adult mouse had abdominal hair removed and was placed in a black box with a cold light source providing trans-illumination (Fig. 8o). The mouse was video recorded (see Table 1 for video specifications) for subsequent EVM processing (color enhanced) while ultrasound imaging was simultaneously used to validate $f_{\mathrm{H}}$ (Additional file 12). Four embryos were positioned in such a way that $f_{\mathrm{H}}$ measurements were possible with both EVM (mean $f_{\mathrm{H}} \pm$ $95 \% \mathrm{CI}=97.40 \pm 8.13 \mathrm{BPM}$ ) and ultrasound (mean
$f_{\mathrm{H}} \pm 95 \% \mathrm{CI}=95.5 \pm 8.09 \mathrm{BPM}$ ) (measurements shown for one embryo in Fig. $8 \mathrm{p}-\mathrm{v}$ ) which was not statistically significantly different $(p=0.52)$ when tested with a paired $t$ test.

\section{Infrasound detection}

Infrasound vocalization $(<20 \mathrm{~Hz})$ in elephants represents a low-frequency, high amplitude signal that is difficult for humans to detect and localize by ear and in theory could be revealed with EVM processing of videographic 


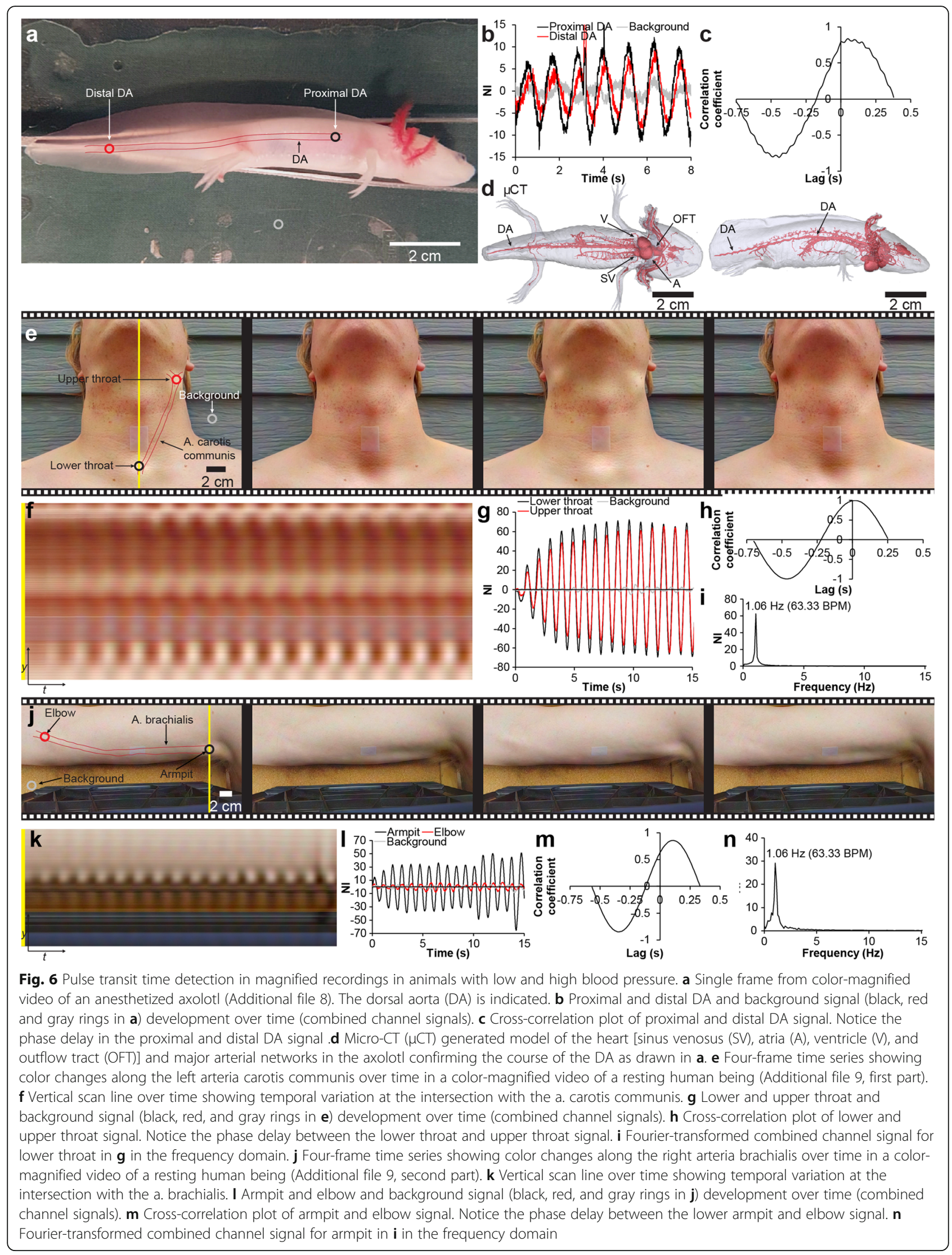



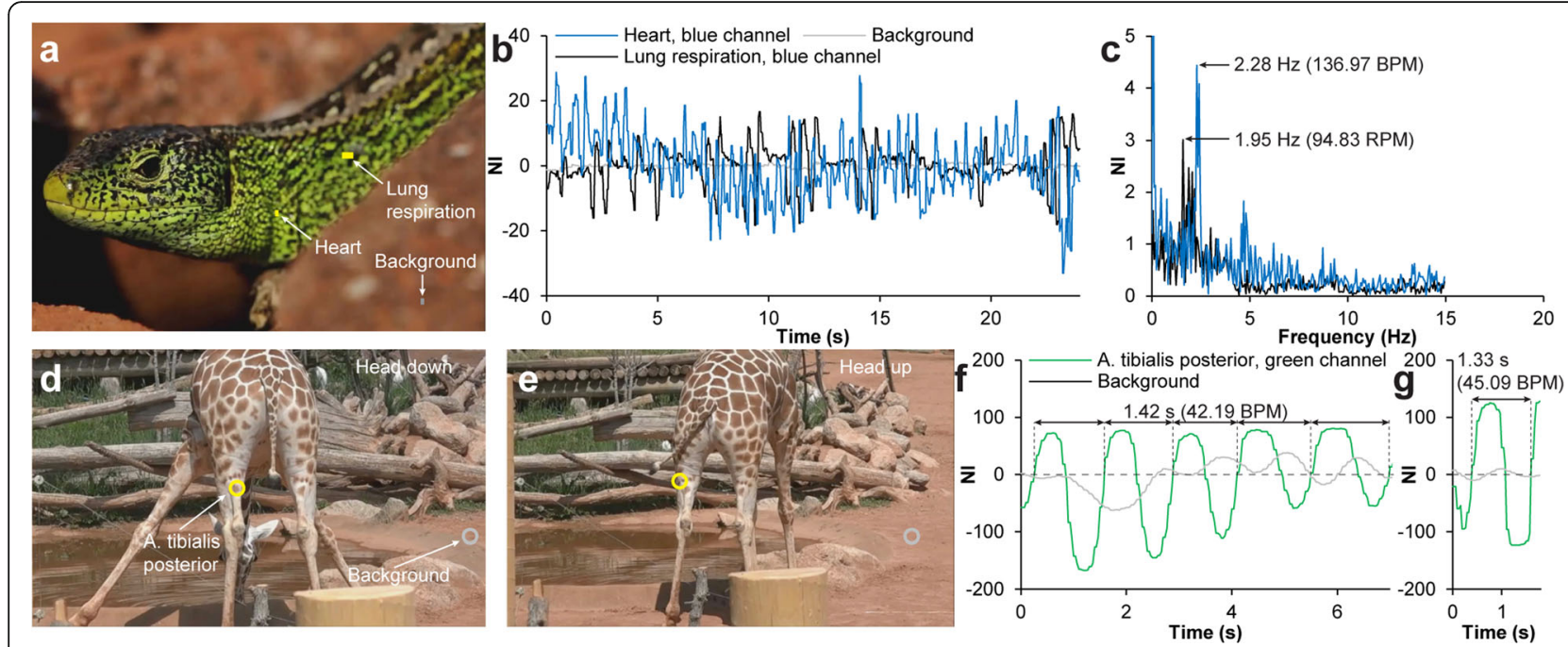

Fig. 7 Signal detection with Eulerian video magnification in non-dedicated videographic material. a Single frame from motion magnified video of sand lizard. Video was located on Flickr and used with permission from Ben Locke. b Heart, lung, and background signal (yellow and gray lines in a) development over time (blue channel). c Fourier-transformed blue channel signal of heartbeat and respiration in $\mathbf{b}$ in the frequency domain. d, e Single-frame color-magnified video of a drinking giraffe (d) that eventually lift its head (e). Video was located on YouTube and used with permission from AliasAnimo. f, $\mathbf{g}$ Arteria tibialis posterior and background signal (yellow and gray rings in $\mathbf{d}$ and e) development over time (green channel). The low number of cardiac cycles in the timespan of the two postures does not allow for meaningful Fourier transformation; thus, heart rate is estimated as an average of the available signal periods for the two postures (5 periods during head down, one period during head up)

material with a modest frame rate ( $>40$ FPS). To test if motion magnification could reveal subtle vibrations on different locations of the body of infrasound vocalizing elephants, we video-recorded three captive Asian elephants (Elephas maximus Linnaeus, 1758), one male (57 years of age) and two females (female 1: 18 years of age, female 2: 18 years of age) of which female 1 was 15 months pregnant at the time of recording. The elephants were video and audio recorded over a period of $931 \mathrm{~s}$ using five separate cameras (Fig. 9a; Additional file 13, first part) each recording with the same spatial resolution $\left(1920 \times 1080 \mathrm{px}^{2}\right)$ and temporal resolutions ranging from 25 FPS to 60 FPS (Table 1, Fig. 9b). During the main part of the recording (from 285 to $768 \mathrm{~s}$ ), the three elephants were positioned in close proximity, facing the closed doorway to the inside enclosure where a caretaker was positioned and communicated with the elephants to encourage them to vocalize. The five cameras were positioned with maximal spacing given the constraints of the fenced enclosure (Fig. 9a-d). Three cameras (CAM 1-3) were stationary and two (CAM 4-5) were manually operated although stabilized on fence poles. During the last part of the recording (768-931 s), the elephant group split up and female 1 walked closely past the cameras. Audio spectrogram analysis revealed 28 distinct vocalization events with an infrasound component (Fig. 9e). Three vocalization events (Fig. 9e: vocalization events $3,4,28$ ) were selected for EVM motion enhancements. Together, vocalization events 3 and
4 represent a sequence of vocalization (event 3 ), silence, vocalization (event 4), and silence (Fig. 10). The video recording made at the lowest temporal resolution (25 FPS, CAM 5) was inadequate for EVM enhancement of infrasound-related vibrations. However, EVM processing of recordings made at higher temporal resolutions (5060 FPS, CAM 1-4) showed a match in time of high amplitude vibrations at the male head and male posterior to the audio spectrogram [compare Fig. 10e, j, o, r (audio spectrograms) with Fig. 10f, k, s (male head EVM processed signal), Fig. 10g, l, t (male head vibration spectrogram), and Fig. 10h, m, p, u (male posterior EVM processed signal); Additional file 13, second part]. On the other hand, there was no match in vibrations observed on the two females and the audio spectrogram [compare Fig. 10e, j, o, r (audio spectrograms) with Fig. 10i, n, q, v (female 1 and 2 posterior EVM processed signal)], suggesting that only the male was vocalizing with an infrasonic component during events 3 and 4 . During vocalization event 28 , female 1 was moving forward and only CAM 4 (manually operated) was in a position to record the entire vocalization event (Fig. 11). The EVM processed recording from CAM 4 showed a very precise match in time of high amplitude vibrations at the head, throat, and posterior to the audio spectrogram [compare Fig. 11d (audio spectrogram) with Fig. 11e, f (female 1 head EVM processed signal and vibration spectrogram), Fig. 11g, h (female 1 throat EVM processed signal and vibration spectrogram), and Fig. 11i (female 1 


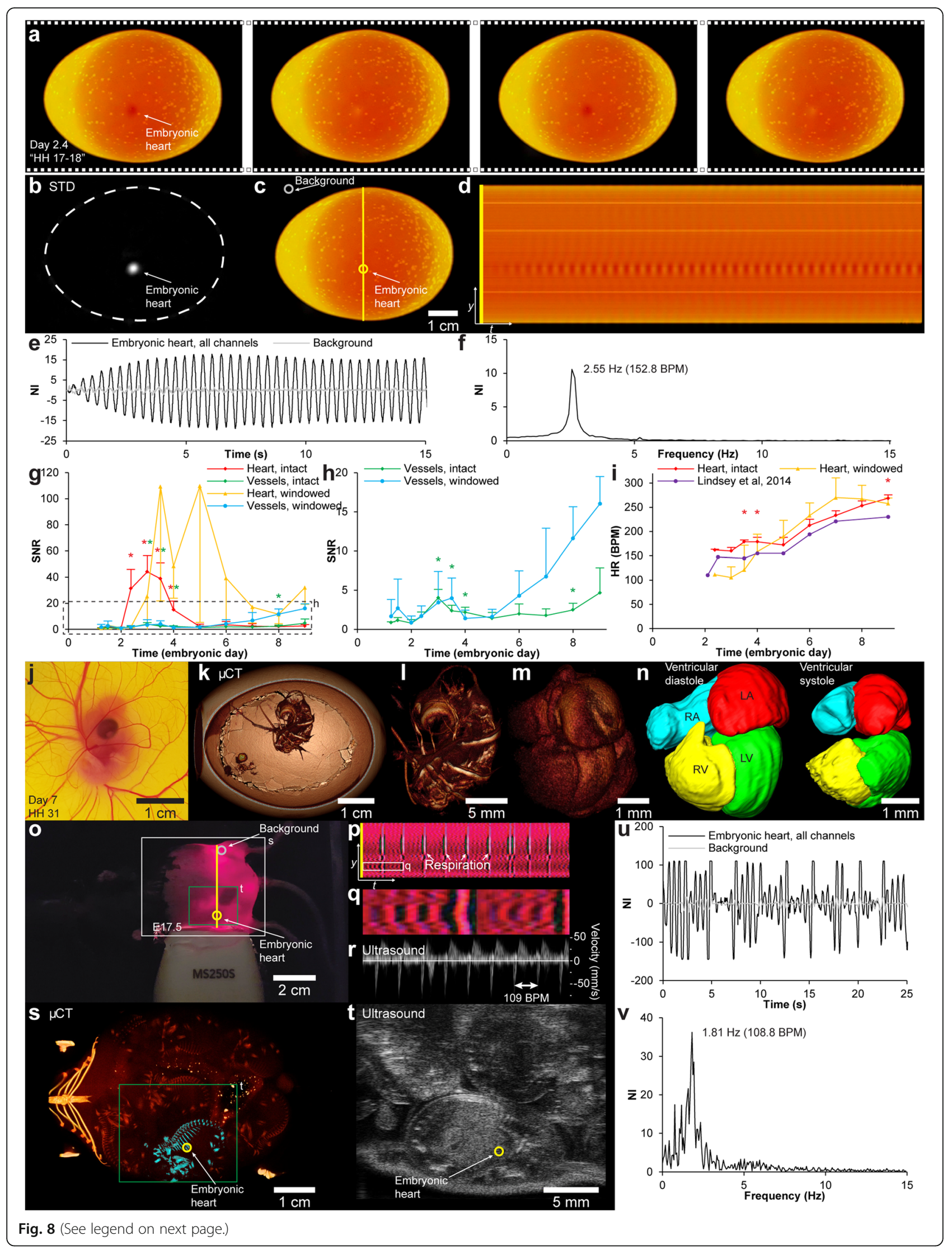


(See figure on previous page.)

Fig. 8 Heart rate detection and acquisition of gating signal in embryonic animals. a Four-frame time series showing color changes associated with the heartbeat in color-magnified video of embryonic chicken at day 2.4 in a candled, non-windowed egg (Additional file 10). b Pixel-wise standard deviation (STD) of magnified video highlighting embryonic heart. c, $\mathbf{d}$ Image probes (c) and vertical scan line over time (d) showing temporal variation at embryonic heart intersection. e, $\mathbf{f}$ Embryonic heart and background signal (yellow and gray rings in c) development over time (e) (combined channels), and Fourier-transformed signal in the frequency domain (f). $\mathbf{g}$ Line chart (mean $\pm 95 \%$ confidence interval) of heartbeat signal to noise ratio (SNR) during 9 days in intact and windowed eggs measured at the embryonic heart or vitelline vessels. $\mathbf{h}$ Enlargement of box in $\mathbf{g}$ (only vessel signals). Color coded asterisks $\left(^{*}\right)$ in $\mathbf{g}, \mathbf{h}$ indicate statistically significant differences from SNR $=1$. $\mathbf{i}$ Line chart comparing heart rate acquired with video magnification to reference values (Lindsey et al., 2014). Asterisks indicate statistically significant differences from reference values. $\mathbf{j}$ Embryonic chicken at day 7. $\mathbf{k}-\mathbf{n}$ Three-dimensional rendering of embryonic chicken at day 7 from micro-CT $(\mu \mathrm{CT})$ imaging gated by magnified optical signal. Volumetric reconstruction of the heart and vascular system of the embryo in a resealed egg $(\mathbf{k}-$ $\mathbf{m}$ ) and segmentation of the heart chambers in two phases (n). o Single frame from color-magnified video of pregnant mouse abdomen (E17.5) (Additional file 12). $\mathbf{p}$ Vertical scan line from magnified video over time. $\mathbf{q}$ Magnification of box in $\mathbf{p}$, showing color fluctuations associated with embryonic heartbeat. $\mathbf{r}-\mathbf{t}$ Ultrasound ( $\mathbf{r}$, pulsed wave Doppler; $\mathbf{t}$, brightness mode) and $\mu \mathrm{CT}$ (s) validation of heart rate and position of embryo. $\mathbf{u}$, $\mathbf{v}$ Embryonic heart and background signal (yellow and gray rings in $\mathbf{o}$ ) development over time (u) (combined channels), and Fourier-transformed signal in the frequency domain $(\mathbf{v})$

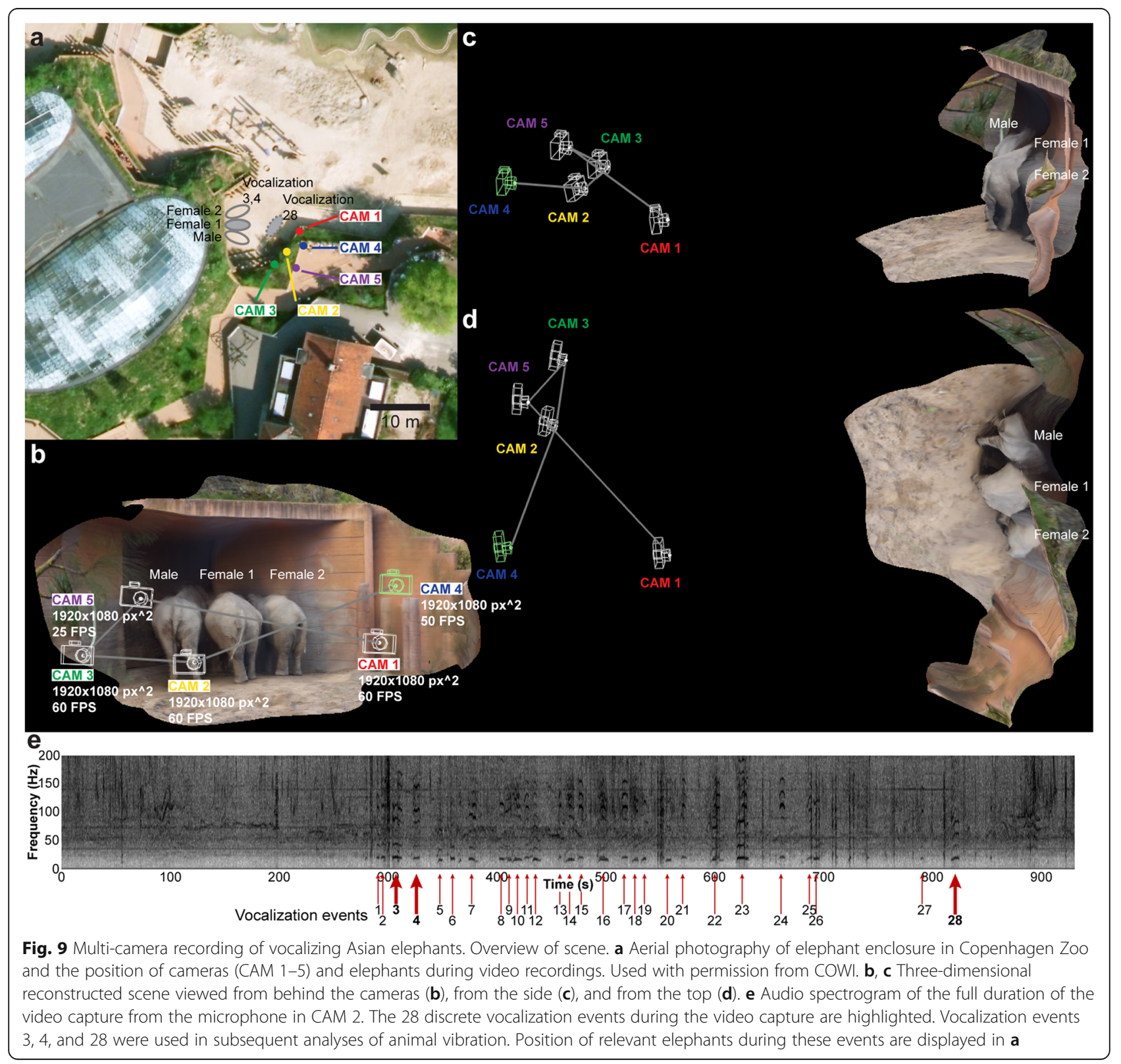



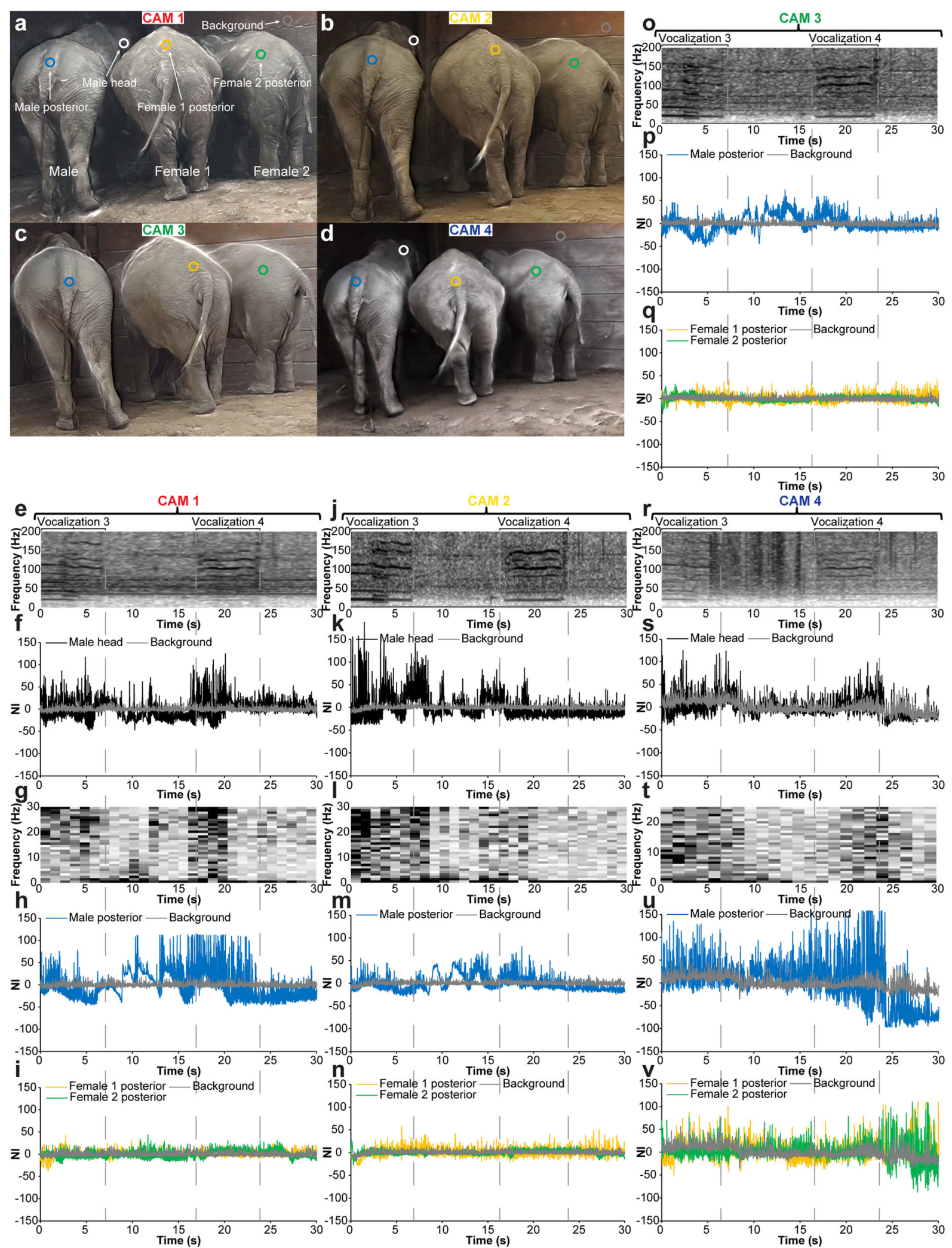

Fig. 10 (See legend on next page.) 
(See figure on previous page.)

Fig. 10 Multi-camera recording of three Asian elephants. Determination of vocalizing elephant with Eulerian video magnification. a-d

Corresponding images from video recordings from CAM 1 (a), CAM 2 (b), CAM 3 (c), and CAM 4 (d). Image probes for vibration analysis are indicated on images and labeled in $\mathbf{a}$. e-v Analysis of vibrations at different body positions of the three elephants during the video capture. The audio spectrograms associated with each recording are displayed at the top $(\mathbf{e}, \mathbf{j}, \mathbf{o}$, and $\mathbf{r})$, and the durations of the two vocalization events (events 3 and 4 in Fig. 9) are highlighted with dashed lines. Plots of normalized pixel intensity (NI) over time for image probes placed on the male head (f, $\mathbf{k}$, and $\mathbf{s}$ ), the male posterior $(\mathbf{h}, \mathbf{m}, \mathbf{p}$, and $\mathbf{u}$ ), the female 1 and female 2 posterior $(\mathbf{i}, \mathbf{n}, \mathbf{q}$, and $\mathbf{v})$ reveal periods with large amplitude vibrations relative to background noise on the surface of the male. The male head was not available for analysis in the video capture by CAM 3 due to the angle of the elephant. By performing a short-time Fourier transform (6-bit window) of the male head signal, vibration spectrograms were generated from the different recordings $(\mathbf{g}, \mathbf{I}$, and $\mathbf{t})$ showing a good match with audio spectrograms $(\mathbf{e}, \mathbf{j}$, and $\mathbf{r})$

posterior EVM processed signal); Additional file 14. In combination, the recordings of vocalization events 3,4 , and 28 demonstrate that EVM could be used to enhance and detect vibrations related to infrasound production at both anterior and posterior positions of the elephant body, and this could be used to determine which animal was vocalizing at a given time.

\section{Discussion}

Eulerian video magnification and other video magnification techniques have been used in several recent studies with a medical scope to extract vital signs from videographic material based on widely available smartphone cameras [31-33], and even a remotely controlled camera mounted on a hovering aerial vehicle (drone) [34]. Additionally, video magnification has been used to enhance detection of fasciculations in people with amyotrophic lateral sclerosis based on videographic material [35] and enhance small motions in ultrasonographic material [36]. Although the medical potential of enhancing subtle color changes or motions in video material is obvious, the potential use of the method in experimental biology and comparative physiology should not be neglected. Thus, the aim of this study was to report how to apply Eulerian video magnification in a more basic experimental biology setting and to provide a series of examples of how signals of relevance, e.g., $f_{\mathrm{H}}$, respiration rate, PWV, and soundinduced vibrations, can be extracted from videographic material.

By examining the axolotl, we establish how several camera formats can be used for EVM processing in a laboratory setting (Fig. 3, Additional file 4) and demonstrate a two-step procedure to optimize band-pass filter and channel selection in the EVM procedure (Figs. 1a and $2 \mathrm{a}-\mathrm{j}$, Additional file 2). For reasons mentioned earlier, the axolotl is a well-adapted model for vital sign monitoring with EVM, and the method demonstrated its potential to extract $f_{\mathrm{H}}$ in both leucistic axolotls as well as in dark brown wild types (Fig. $4 \mathrm{a}-\mathrm{C}$ ) and in both single animal and multiple animal setups (Figs. 1, 2, 3, and 4, Additional files 1, 2, 3, 4, 5, 6 and 8). The simple physiology experiment in which $f_{\mathrm{H}}$ was measured with EVM under different physiological conditions (rest, exercise and anesthesia) demonstrates a laboratory setup in which $f_{\mathrm{H}}$ can be continuously monitored solely based on a camera signal, which in experimental cases of little or predicted variance in oxygen pulse, allows for a constant indirect evaluation of $M R$ [18].

Under field conditions, video recording can be a useful method to document animal behavior. Heart and respiration rate may be welcome additional pieces of physiological information that, in some cases, can be extracted from videographic material. Whereas this possibility can come into consideration in prospective planning of future experimental setups, we also demonstrate that EVM can be used retrospectively on videographic material that was not produced with the intention of video magnification (Fig. 7). This can have relevance for comparative ecophysiology studies that require $f_{\mathrm{H}}$ measurements or $M R$ estimations on non-disturbed animals in their natural environment. Given the availability of high-quality videographic material of wild animals that already exists in the form of nature and wild-life documentaries, informative studies may be made using EVM.

The selection of species that were used in the experimental series of this study, axolotl, zebrafish, human, sand lizard, giraffe, embryonic chicken, embryonic mouse, and elephant, reveal an important consideration for evaluating appropriateness of different animals for EVM, namely the lack of thick fur or plumage on the entire body surface or on select parts allowing for unobstructed view to the skin surface.

Measurements of $f_{\mathrm{H}}$ acquired with EVM in this study were validated, where possible, with other techniques (in axolotl and embryonic mouse with ultrasound, in human with palpation, and in embryonic chicken with direct observations of windowed eggs). Validation was not possible in the zebrafish, since any human interaction changes behavior and most likely $f_{\mathrm{H}}$ in this observant fish (personal observation), and electrocardiography (ECG) was inapplicable in an aquarium with multiple animals. However, the obtained $f_{\mathrm{H}}(\mathrm{EVM}) \pm 95 \% \mathrm{CI}=$ $127.57 \pm 7.87 \mathrm{BPM}$ in the adult zebrafish corresponds well with, to our knowledge, the only other literature account of non-invasive measurement of conscious adult zebrafish resting $f_{\mathrm{H}}$ of 135-140 BPM in fish of the age of 50-100 days at $28^{\circ} \mathrm{C}$ [37]. In this previous study, $f_{\mathrm{H}}$ was 


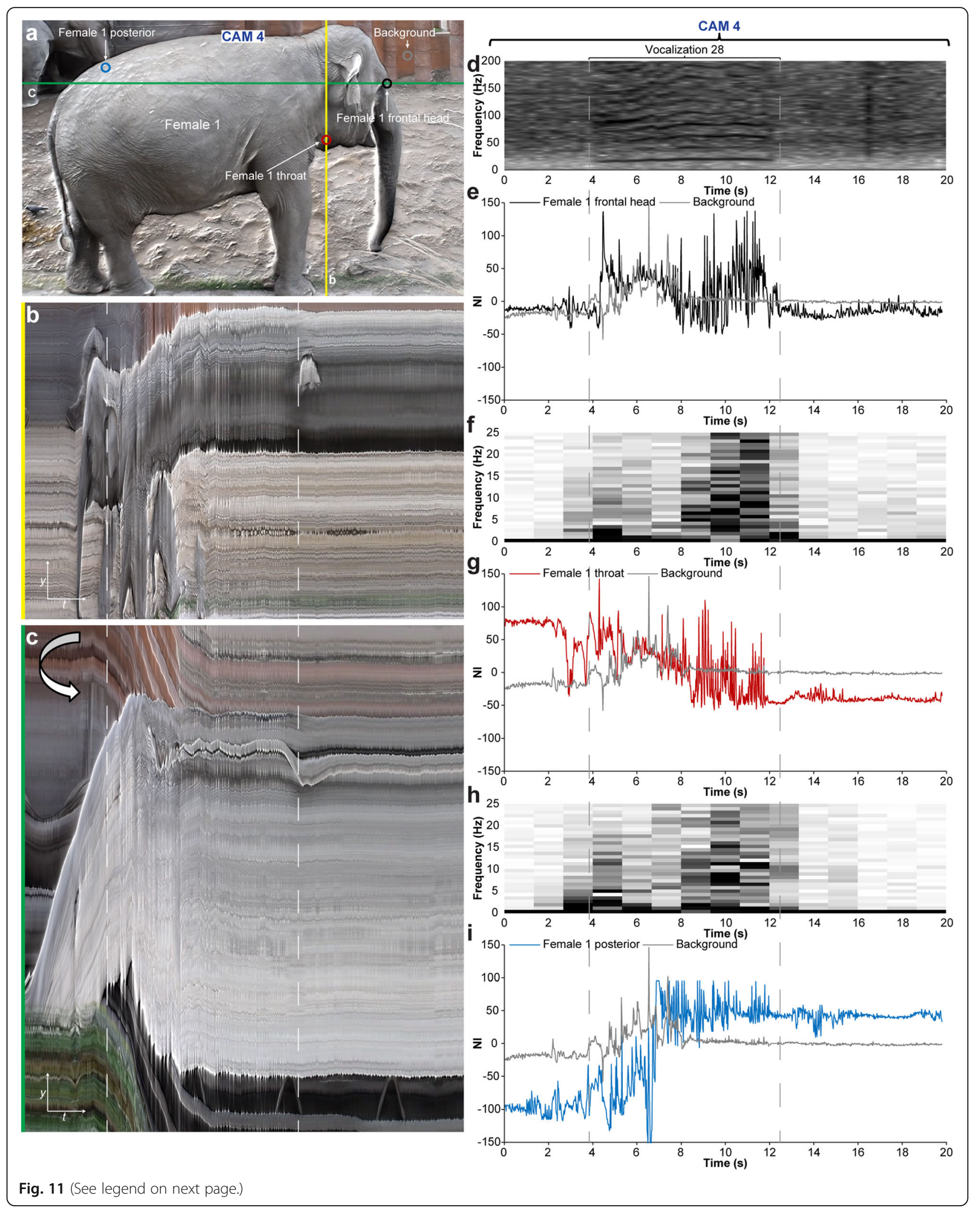


(See figure on previous page.)

Fig. 11 Single close-up video recording of one Asian elephant showing pronounced vibrations associated with vocalization. a Image from video recording by CAM 4. Image probes for vibration analysis are indicated on the image. b, c Vertical (b, yellow line in $\mathbf{a}$ ) and flipped horizontal (c, green line in a) scan lines plots over time. The duration of one vocalization event (event 28 in Fig. 9) is highlighted by dashed lines. $\mathbf{d}$ Audio spectrogram. e-i Analysis of vibrations at different body positions during the video capture. Plots of normalized pixel intensity over time at the frontal head (e), throat $(\mathbf{g})$, and posterior (i). f, $\mathbf{h}$ Vibration spectrograms from frontal head and throat signal. By performing a short-time Fourier transform (6-bit window) of the frontal head and throat signal, vibration spectrograms were generated ( $\mathbf{f}$ and $\mathbf{h})$ showing a good match with audio spectrogram (d)

acquired by trans-illuminating individually isolated fish with a powerful light source, which could influence resting $f_{\mathrm{H}}$ via stress effects on autonomic $f_{\mathrm{H}}$ control. Thus, to the best of our knowledge, the present study provides the first account of post absorptive and undisturbed $f_{\mathrm{H}}$ in conscious, unrestrained and undisturbed adult zebrafish under normal light conditions and social setting of the animal. Although movements can be detrimental to EVM processing of videographic material, the zebrafish recordings demonstrate that even with rapid movements occurring at a high rate (Fig. $5 \mathrm{f}$ ) it can be possible to extract $f_{\mathrm{H}}$ if this is out of the frequency range of the movements, and these displacements are small compared to the size of the animal.

Heart rate validation was also not possible in videographic material acquired from online sources that was not produced with the purpose of video magnification and $f_{\mathrm{H}}$ measurement (Fig. 7). The sand lizard (Fig. 7a-c) represents an ectotherm; thus, $M R$ and $f_{\mathrm{H}}$ is highly dependent on ambient temperature and direct comparison with acquired $f_{\mathrm{H}}$ under unknown circumstances with literature derived $f_{\mathrm{H}}$ is problematic for this species. This point highlights an important consideration in designing EVM studies, namely the importance of initially ground truthing EVM processed video recordings of parameters of interest such as $f_{\mathrm{H}}$ and respiration rate with other methods such as ultrasound, direct observations or at least expected frequency ranges based on literature. The giraffe (Fig. $7 \mathrm{~d}-\mathrm{g}$ ) on the other hand is endothermic, and under the assumption that the filmed animal is healthy and unstressed, the measured $f_{\mathrm{H}}$ of $42-45 \mathrm{BPM}$ falls close to previously reported values of about $40 \mathrm{BPM}$ in undisturbed quietly standing giraffes [38-40]. It has long been a topic of discussion how the giraffe regulates cerebral pressure and blood flow when changing posture from a head down situation (typically drinking) with the brain some $2 \mathrm{~m}$ below the heart to a head up situation with the brain $2-3 \mathrm{~m}$ above the heart in the matter of seconds and vice versa [39]. In a sophisticated experiment, Brøndum et al. [39] found that raising and lowering the head of anesthetized giraffes caused substantial changes in the blood flow and pressure in blood vessels of the neck but no significant change in $f_{\mathrm{H}}$ [mean $f_{\mathrm{H}}$ (head down) \pm standard error of mean (SE) $=42 \pm 4$ $\mathrm{BPM}$; mean $f_{\mathrm{H}}$ (head up) $\left.\pm \mathrm{SE}=43 \pm 4 \mathrm{BPM}\right]$. Although the measurements acquired with EVM of the video of a giraffe with the head down and up in this study support this observation (Fig. $7 \mathrm{~d}-\mathrm{g}$ ), the data material for the head up posture (a single cardiac cycle) is too sparse to draw concrete conclusions.

In addition to $f_{\mathrm{H}}$ detection, we also demonstrate how EVM can be used to measure PWV in both a low blood pressure system such as the axolotl (Fig. 6a-d) and a high-pressure system such as the human being (Fig. 6e$\mathrm{n})$. To the best of our knowledge, no literature values for PWV in the axolotl dorsal aorta exist; however, the measured PWV in the human being [PWV (a. carotis communis $)=4.38 \mathrm{~m} / \mathrm{s} ; \quad \operatorname{PWV}($ a. brachialis $)=3.62 \mathrm{~m} / \mathrm{s}]$ are well within human reference values for PWV in the a. carotis communis and a. brachialis [41-43]. The acquisition of PWV from non-contact videographic material is interesting in a clinical setting since, for large elastic vessels, PWV both correlates non-linearly with arterial stiffness and can be applied to measure this important clinical parameter using the Moens-Korteweg equation, and PWV also correlates non-linearly with blood pressure, and thus, changes in PWV can be used to derive relative changes in blood pressure [44]. To obtain absolute blood pressure values, a subject-specific calibration is needed [43]. The applicability of EVM to acquire precise and accurate indirect measurements of blood pressure remains to be shown. However in this task, it is important to take account of the fact that the correlation of the two parameters is not valid in peripheral nonelastic vessels; thus, a previous attempt to measure pulse transit time with EVM at the neck and at the wrist of a bent arm [45], i.e., a span of vessels that contain peripheral non-elastic arteries, would not allow for PWV conversion to blood pressure.

The mid-late stages of embryonic heart development in birds and mammals represent an important timespan for the formation of structures that characterize highperformance four-chambered hearts. During this time, cushions form that later mature into valve leaflets and septation results in separate atria and heart chambers [30]. To understand what factors affect correct and incorrect heart development which can lead to congenital heart defects, it is important to be able to image the developing hearts of well-established avian and murine models at these stages. Micro-CT imaging provides a 
method to in vivo image mid-late-term developing hearts in chicken and in mouse, in direct threedimensional acquisitions, in contrast to high-frequency ultrasound that currently only exists with linear array (2D) transducers, at a higher resolution than magnetic resonance imaging allows for, and with a larger depth of field than possible with optical coherence tomography. However, the minute beating hearts of the embryonic chicken and mouse are challenging to image since X-ray projections need to be allocated to specific cardiac phases to avoid image blurring. Electrocardiography can be applied to embryonic chicken [46]; however, electrode placement close to the embryo can result in image artifacts, and for the embryonic mouse, it is difficult to discriminate between the ECG from several embryos. We have previously used a non-enhanced optical signal acquired at the level of the beating heart or oscillating proximal vessels to prospectively gate micro-CT scans of chicken embryos at embryonic days 9 and 11 in windowed eggs (unpublished data). Here, we demonstrate that the less intense heartbeat signal at embryonic day 7 can be magnified to a level where it is detectable even through the eggshell, allowing for prospectively gated data acquisition with the micro-CT system (Fig. 8k-n, Additional file 11). Likewise, the heartbeat signal in embryonic mice in utero was detectable after EVM processing (Fig. 8o-v, Additional file 12), in theory allowing for a similar optical gating procedure of a micro-CT system if a protocol to administer a vascular micro-CT contrast agent to the embryonic mouse in utero is developed.

Vocalization in mammals span a frequency range of 9 $\mathrm{Hz}$ in blue whales [Balaenoptera musculus (Linnaeus, 1758)] [47] to $110 \mathrm{kHz}$ in some bats [48]. Sound is a mechanical wave, and in those cases where sound production results in vibrations on the body surface of the source, these small motions can, in theory, be magnified in videographic data with EVM without aliasing, given that the data material has a temporal resolution of twice the frequency of the sound (Nyquist-Shannon sampling theorem), i.e., 18-220,000 FPS. In comparison, even the highest heart rates recorded in vertebrates, 1260 BPM $(21 \mathrm{~Hz})$ in blue-throated hummingbird [Lampornis clemenciae (Lesson, 1829)] [49] and 1511 BPM $(25.18 \mathrm{~Hz})$ in Etruscan shrew [Suncus etruscus (Savi, 1822)] [50], could in theory be video magnified with EVM without aliasing on videographic material with a modest frame rate just above 50 FPS. Thus, inexpensive camera equipment allows for $f_{\mathrm{H}}$ detection across all vertebrates that may be applicable for EVM, but without dedicated highspatiotemporal resolution camera equipment, the video magnification of sound-induced vibrations is restricted to animals that vocalize at low frequencies with high amplitudes. All three extant species of elephants, the Asian elephant, the African bush elephant [Loxodonta africana (Blumenbach, 1797)], and the African forest elephant [Loxodonta cyclotis (Matschie, 1900)] vocalize and communicate with infrasound with fundamental frequencies typically in the range of $14-35 \mathrm{~Hz}$ with sound pressure levels as high as $103 \mathrm{~dB}(\mathrm{re} 20 \mu \mathrm{Pa})$ at $5 \mathrm{~m}$ from the source [51-54]. Infrasound production allows for long range communication between elephants, and it has been shown that the fundamental frequency of a rumble conveys information about the elephant that produced the sound, e.g., rumbles of females exhibit higher fundamental frequencies as compared to male rumbles [55], age is negatively correlated with fundamental frequency $[56,57]$, and individuals experiencing elevated levels of affect produce rumbles of a higher fundamental frequency $[58,59]$. It has been demonstrated that myoelastic-aerodynamic flow-driven sound production can account for loud infrasonic vocalization in the African bush elephant without the need of active muscular contraction (purring) mechanisms [60]. Both mechanisms, however, would result in potentially detectable oscillations originating from the throat/head region propagating on the surface of the elephant body and possibly being attenuated at distal positions. In behavioral studies, it may be of interest to discern between individual vocalizing elephants at any given point in time. Although acoustic triangulation can be used in the field to localize a vocalizing elephant $[61,62]$, this method falls short when individual vocalizing elephants are in close proximity because of measurement error. Simple observation is frequently inadequate because calls often are not audible and vocalizations are not always linked to visible behaviors. In vocalization events 3-4 presented in this study, we were able to demonstrate, using multiple cameras, vibrational signals in the frequency range of infrasound with different intensities from three Asian elephants standing in close proximity in a suboptimal position for throat/head produced vibration detection (rear of animals facing the cameras, Fig. 9). Signal intensity was several folds higher in the male than the two females suggesting that the infrasonic component of the vocalization originated from the male in the group (Fig. 10, Additional file 13). Whether EVM is complementary to acoustic localization arrays for localization of a vocalizing elephant in a group in long range field conditions needs to be explored further using highresolution cameras and high power telescopic lenses. In vocalization event 28 presented in this study, a single elephant was recorded vocalizing in close proximity to the camera (Fig. 11). A pronounced vibrational signal with a precise match in time with the acoustic signal could be detected with EVM both in the throat/head region and in the posterior region. No signal attenuation was observed between the proximal and distal locations (Fig. 11) suggesting that the vibrational signal travels 
well in the massive body of the elephant, meaning that the body position relative to the camera is of less importance when enhancing the subtle sound-induced surface vibrations in vocalizing elephants.

\section{Conclusions}

In this methodology study, it is demonstrated how EVM can be used to acquire $f_{\mathrm{H}}$, respiration rate, pulse wave velocity, and infrasound vocalization-related vibrations from videographic material in a range of experimental setups. The method does not generate a signal where there is none, but enhances rhythmic signals, such as the heartbeat, relative to the noise level, so an inapparent signal can be localized, visualized, and recorded. The EVM methods work across a wide range of video qualities and spatiotemporal resolutions. Optimally, test objects are stationary, but movements out of the frequency range of the signal of interest can be acceptable. The importance of physiological measurements from undisturbed animals in their natural environment is becoming ever clearer in the fields of experimental biology and ecophysiology, which may in some cases limit the applicability of instrumentation of animals with recording devices. In this respect, EVM can, after a thorough validation with other techniques, provide a tool to prospectively or retrospectively extract signals of interest from a distance using simple inexpensive video recording equipment.

\section{Methods}

\section{Animals and husbandry}

Animals used for laboratory-based experiments in this study were the Mexican axolotl, the zebrafish, embryonic chicken, and mouse. Axolotl and zebrafish experiments were performed in Denmark and were approved by the Danish National Animal Experiments Inspectorate (Protocol\# 2015-15-0201-00615 and Protocol\# 201915-0201-00091, respectively). Axolotls were obtained from a commercial breeder (Exoterra $\mathrm{GmbH}$, Germany) and were housed individually in plastic containers with a $10-\mathrm{cm}$ water depth and a $930-\mathrm{cm}^{2}$ surface area with regular water changes and a $12 \mathrm{~h}: 12 \mathrm{~h}$ light to dark cycle. Animals were fed every second day with proteinenriched trout pellets. Axolotl anesthesia was obtained using $200 \mathrm{mg} / \mathrm{L}$ ethyl-4-aminobenzoate. Zebrafish were obtained from a commercial breeder (Lystrup Dyrecenter, Denmark) and were housed initially 61 animals together in a large 200-1 aquarium with environmental enrichment via grating, brick, and stones; stocking density at the time of recording was 45 fish in the 200-1 aquarium. Aquarium air stones provided aerated water, a thermostatic heating system set at $28^{\circ} \mathrm{C}$ providing a measured temperature of $27.6{ }^{\circ} \mathrm{C}$, and pump (EHEIM 200 , Germany) with a $20-\mathrm{cm}$ water depth and $4500-\mathrm{cm}^{2}$ surface area with regular water changes and a $12 \mathrm{~h}: 12 \mathrm{~h}$ light to dark cycle. Animals were fed daily with JBL NovoBel (tropical aquarium fish food, JBL, GmbH \& Co.KG, Germany). Embryonic chicken experiments were performed in the USA (NY) where Institutional Animal Care and Use Committee (IACUC) approval does not apply for embryonic chickens. Eggs were obtained from local commercial breeder and were incubated with rocking at $37.5^{\circ} \mathrm{C}$ in a water-saturated environment. All chicken embryos were terminated at embryonic day 9 . The embryonic mouse experiment was performed in the USA (NY) where mice were bred at the lab animal facility under IACUC protocol \#2008-0011. Mouse anesthesia was obtained by isoflurane inhalation (4\% isoflurane in pure $\mathrm{O}_{2}$ for induction of anesthesia and 1.5\% isoflurane in pure $\mathrm{O}_{2}$ for maintaining anesthesia), and the adult mouse was euthanized under anesthesia directly after video recording.

\section{Video material, camera, and audio}

All video material from lab-based experiments was recorded using the built-in camera in a standard smartphone (Galaxy S6, Samsung, Seoul, South Korea) or a GoPro Hero 4 (GroPro Inc., San Mateo, CA, USA) compact camera applying spatiotemporal resolution settings as specified in Table 1. For the multi-camera recording of vocalizing elephants, the camera and built-in microphone of three different smartphones [Galaxy S6, Galaxy S6 Edge, and iPhone 6s (Apple Inc., Cupertino, CA, USA)] and two digital single-lens reflex cameras (Nikon D5300 with a AF-S DX NIKKOR 18-55 mm f/3.5-5.6G VR II lens and Canon Eos 300D with a Canon EFS 18$55 \mathrm{~mm} \mathrm{f} / 3.5-5.6 \mathrm{G}$ Image stabilizer lens) were used.

Video material that was not produced with the intention of EVM processing was acquired from external online sources. The sand lizard video was produced by Ben Locke and was located on Flickr [63]; the giraffe video was produced by AliasAnimo and was located on YouTube [64]. Written permissions were obtained to apply the videos for EVM processing in the scope of the present study.

\section{Ultrasonography and micro-CT imaging}

Ultrasonography on axolotl and embryonic mouse was performed with a Vevo 2100 system (FUJFILM VisualSonics, Toronto, Ontario, Canada) with a $21-\mathrm{MHz}$ transducer (MS250).

Ex vivo micro-CT imaging of a contrast agent (Microfil, FlowTech Inc., Carver, MA, USA) perfused axolotl was performed using a Scanco Medical XtremeCT system (Scanco Medical AG, Brüttisellen, Switzerland) with 1500 projections $/ 180^{\circ}$ and an isotropic voxel size of $41 \mu \mathrm{m}$, an X-ray tube voltage of $59.4 \mathrm{kVp}$, an X-ray tube current of $119 \mu \mathrm{A}$, and an integration time of $132 \mathrm{~ms}$. In 
in vivo micro-CT imaging of embryonic chicken, an ex vivo imaging of embryonic mouse was performed using a eXplore CT 120 (GE Healthcare, Chicago, IL, USA) system with 1200 projections $/ 180^{\circ}$ and an isotropic voxel size of $25 \mu \mathrm{m}$, an X-ray tube voltage of 80 $\mathrm{kVp}$, an X-ray tube current of $30 \mu \mathrm{A}$, and an integration time of $30 \mathrm{~ms}$. For the in vivo embryonic chicken scans, an endoscopic camera (Qyuhe Mini USB Endoscope) was positioned in such a way that the egg surface could be viewed without the endoscope overlapping the trajectory of the X-ray beam of the micro-CT scanner. A flashlight placed directly at the end of the egg provided light for candling and heat during the micro-CT acquisition. Prospective gating was applied via custom-made peak detection software processing the real-time EVMenhanced signal from the endoscope and signaling the micro-CT system a $5 \mathrm{~V}$ transistor-transistor logic false ECG pulse via a Bayonet Neill-Concelman connector.

\section{Eulerian video magnification procedure}

The Matlab source code to perform EVM was released along with the original report of the procedure [10], and can be downloaded and used for research purposes from [65]. Additionally, an online freeware version exists for simple magnification procedures [66] as well as a commercial application package [66] and a software development package [66]. To demonstrate the relatively easy application of EVM in experimental biology, all videos in this study were processed using either the online freeware tool or the application package. To perform real-time EVM, the application package was needed. The EVM procedure of all videos in the study is outlined in the block diagram in Fig. 1a. Initially, a wide passband (frequencies of interest), a magnification type (color or motion magnification), and magnification level were selected. The resulting output video with a low SNR was used to extract an initial oscillation frequency, and this information was used to narrow the band-pass filter and reprocess the recording to produce a magnified video with a higher SNR. Frames were extracted from magnified videos using QuickTime Player version 7.7.9 (Apple Inc., Cupertino, CA, USA) and saved as three-dimensional stacks in TIF-format. Image analysis, i.e., channel splitting of RGB videos, selection of regions of interest, and signal extraction, was performed in Image J 1.5e (National Institutes of Health, USA). Unless otherwise specified, signal plots throughout figures are presented as normalized signal intensity (NI, i.e., signal intensity at any given time minus average signal intensity over the entire recording) over time or frequency. To decompose the signal into its constituent frequencies, Fourier transformation was used.

The most important step in the EVM procedure is the acquisition of an input video of adequate quality for subsequent processing. A list of prioritized considerations in the acquisition of videographic material for EVM or evaluation of non-EVM-dedicated material for the procedure is provided below:

1. Stable camera. A stable camera setup is allimportant when recording videographic material for EVM processing. A moving camera results in a moving video frame, resulting in magnification of movements of all surfaces in the recording. Voiceoperated cameras or delayed recording after touching the camera can be useful.

2. Constant background. The magnification technique does not discriminate on objects in focus and those in the background. Therefore, the background in the video should optimally be non-moving and preferably uniform in color.

3. Reflections, shadows, and light source. Reflection and shadows can interfere with EVM processing. If the experimental setup allows for it, reflections and shadows should be kept at a minimal level by adjusting the light source and structures in the vicinity of the scene. Some light sources oscillate with the utility frequency of the power grid, usually 50 or $60 \mathrm{~Hz}$ depending on location. For EVM processing in this frequency range, non-oscillating light sources should be used.

4. Non-moving animal. Eulerian video magnification is easiest to implement on non-moving animals. However, if movements appear at a frequency out of the frequency range of interest (e.g., the slow movement of an elephant producing infrasound at a higher frequency, Fig. 10 and Additional file 13), magnification can still be applicable.

5. Choice of camera. Eulerian video magnification can be used to videographic material produced on most currently available digital cameras. In this study, we primarily used smartphone cameras to demonstrate that the technique works with widely available camera type.

6. Temporal resolution of the video (frame rate). When trying to acquire an analog signal with a digital recorder, the frequencies that can correctly be obtained need to be below the Nyquist frequency, i.e., half of the sampling frequency to avoid aliasing (overlap of the signal). For example, a video recorded at 30 FPS one can only correctly magnify vibrations with a frequency $<15 \mathrm{~Hz}$. Vibrations at higher frequencies may still be magnified so that they are visible on the magnified video, but frequency detection is unreliable in an undersampled system.

7. Spatial resolution of the video and color depth. High-resolution, large color depth videos are 
desirable when trying to magnify subtle color changes or faint vibrations. Still, relatively lowresolution videographic material may still be useful [e.g., the low-resolution recording $\left(640 \times 480 \mathrm{px}^{2}\right)$ in Fig. $3 \mathrm{u}$ and Additional file 4 (last part) still allows for heartbeat magnification].

8. Distance to object and optics. Optimally, the object of interests should be close to the camera to detect small color changes and vibrations. Optics such as telescopic lenses can be used.

9. View point. It is important to consider the camera viewpoint of the object of interest. Some signals may be best observed from specific angles (e.g., a faint heartbeat signal is easier to detect from the ventral surface rather than the dorsal surface of most animals). In some cases, the experimental setup may dictate possible viewpoints that then need to be tested for the applicability with EVM.

10. Duration of video. Eulerian video magnification is a relatively processor demanding technique; therefore, the duration of videographic material should be limited to avoid unmanageable data acquisition.

\section{Statistics}

Relevant types of $t$ tests (paired/unpaired, one/twotailed) were used to test for statistically significant differences between two groups. Relevant types of ANOVAs (repeated measure/mixed model, one-way/ two-way) were used for omnibus testing of statistically significant difference between more than two groups. For post hoc tests of difference between groups, Tukey Honestly Significant Difference tests or Bonferroni corrected $t$ tests were used. Significance level $(\alpha)$ was set at 0.05 unless specified in the case of the Bonferroni correction. For all statistical tests, $p$ value was reported as actual number unless it was smaller than $\alpha / 5$ in which cases it was reported as $p<\alpha / 5$. Error bars in figures represent $95 \%$ confidence intervals.

\section{Supplementary information}

Supplementary information accompanies this paper at https://doi.org/10. 1186/s12915-019-0716-7.

Additional file 1. Simultaneous video recording for Eulerian video magnification and echocardiography. First two parts are dual trace videos of anesthetized leucistic axolotl. Original video to the left and Eulerian Video Magnified video to the right. First part shows color magnification, second part shows motion magnification. Third part is brightness mode echocardiographic video recorded simultaneously to filming. Note that all parts have a sound trace originating from pulsed wave Doppler ultrasound recorded simultaneously, revealing a match in heart rate with color changes and motion in the thoracic and gill region. Video relates to Fig. $1 \mathrm{~b}$-i.
Additional file 2 Effect of band-pass filter size on Eulerian video magnified material. Eulerian Video Magnified (color) recordings of anesthetized leucistic axolotl processed with a wide band-pass filter (first part) and a narrow band-pass filter (second part). Note the pronounced noise reduction when processing with a narrow band-pass. Video relates to Fig. 2a-f.

Additional file 3. Heartbeat magnification under three different physiological conditions using Eulerian video magnification. Eulerian Video Magnified (color) recordings of one leucistic axolotl at different physiological conditions. First part at rest, second part after exercise, third part under anesthesia. Note the frequency difference of color changes at the gills i.e. difference in heart rate under the different conditions. Video relates to Fig. $2 k-x$.

Additional file 4. Effect of spatiotemporal resolution on Eulerian video magnified material. Eulerian Video Magnified (color) recordings of anesthetized leucistic axolotl at six different spatiotemporal resolutions. All resolutions allowed for heartbeat signal magnification and rate detection. Video relates to Fig. 3.

Additional file 5. Heartbeat magnification in colored animal using Eulerian video motion magnification. Eulerian Video Magnified (motion) recording of one brown wild type axolotl axolotl. Dual trace video. Original video to the left and motion magnified video to the right. Note subtle movements of the front limbs in relation to the heartbeat. Video relates to Fig. 4a-c.

Additional file 6. Heartbeat magnification in multi-animal setup using Eulerian video magnification. Eulerian Video Magnified (color) recording of six leucistic axolotls at rest. Video relates to Fig. 4d-k.

Additional file 7. Heartbeat magnification in conscious, unconstrained zebrafish using Eulerian video magnification. Eulerian Video Magnified (color) recording of wild type conscious and unconstrained adult zebrafish. Although the fish is moving slightly during the recording resulting in an increased level of noise particularly in the caudal part of the fish, a heartbeat signal can be extracted from the ventral thoracic surface. Video relates to Fig. 5.

Additional file 8. Pulse wave transit time detection in low blood pressure animal using Eulerian video magnification. Eulerian Video Magnified (color) recording of one anesthetized leucistic axolotl. Pulse wave propagation along the dorsal aorta can be detected at different positions allowing for a pulse transit time measurement. Video relates to Fig. 6a-c.

Additional file 9. Pulse wave transit time detection in high blood pressure animal using Eulerian video magnification. Eulerian Video Magnified (color) recordings of resting human being. Dual trace video. Original video to the left and color magnified video to the right. First part shows color magnification on the throat demonstrating pulsations in the arteria carotis communis, second part shows color magnification on the upper arm demonstrating pulsations in the arteria brachialis. Pulse wave propagation along the arteries can be detected at different positions allowing for a pulse transit time measurement. Video relates to Fig. 6d-m.

Additional file 10. Heartbeat magnification in embryonic chicken using Eulerian video magnification. Eulerian Video Magnified (color) recording of fertilized chicken egg at day 2.4 of embryonic development. The embryonic heartbeat can be detected through the eggshell of the candled egg. Video relates to Fig. 8a-f.

Additional file 11. Interactive two-phase model of embryonic chicken heart at day 7 of development. Three-dimensional interactive model of beating embryonic chicken heart at day 7 of development in two phases: Ventricular end-diastole and ventricular end-systole. The interactive PDF file should be viewed in Adobe Acrobat Reader 9 or higher. To activate the $3 \mathrm{D}$ feature click the model. Using the cursor it is now possible to rotate, zoom, pan the model, and in the model tree all segments of the model can be turned on/off or made transparent. The model tree is a hierarchy containing several sub layers that can be opened (+). Model relates to Fig. 8n.

Additional file 12. Heartbeat magnification in embryonic mouse using Eulerian video magnification. Eulerian Video Magnified (color) recording 
of pregnant mouse with 10 embryos at day 17.5 of embryonic development. The embryonic heartbeat of some embryos can be detected in utero of the candled mother. Video relates to Fig. 8o-v.

Additional file 13. Eulerian video magnification of vibrations related to infrasound vocalization in Asian elephants. Overview of scene and magnified video recordings of three elephants. First part of the video provides an overview of the scene via an aerial photograph (used with permission from $\mathrm{COWI}$ ) and by a three-dimensional reconstruction of the scene. In the second part four synchronous recordings of vocalizing elephants (vocalizing event 3 and 4) from CAM 1-4 that have been video magnified (motion) are displayed. Note that the recordings from each camera have a sound trace in which upper harmonics of low frequency vocalization are audible. Video relates to Figs. 9 and 10 .

Additional file 14. Eulerian video magnification of vibrations related to infrasound vocalization in Asian elephant. Magnified video recording of one elephant close to camera. Video magnified recording of vocalizing elephant (vocalization event 28). Vocalization results in pronounced vibrations of the elephant (4-12s in the video). Note that the video has a sound trace in which upper harmonics of low frequency vocalization are audible. Video relates to Fig. 11.

\section{Acknowledgements}

We wish to acknowledge Ablajan Mahmut, Veer Vekaria, and George Benjamin Voss for help with embryonic mouse imaging; Brian T. Sønderborg, Angelina Kalaba, and Allan Oliver for help with elephant filming; Warren R. Zipfel for help with micro-CT gating; and Heidi Meldgaard Jensen for animal care for the zebrafish.

\section{Authors' contributions}

$H L, M P$, and JTB conceived the study. HL performed the axolotl experiments. $\mathrm{HL}$ and CJAW performed the zebrafish experiments. HL and SG performed the chicken experiments. HL performed the mouse experiments. $\mathrm{HL}$ performed the human experiments. HL and MFB performed the elephant experiments with guidance from $\mathrm{KLP}, \mathrm{DH}$, and PHW. $\mathrm{HL}$ drafted the manuscript with guidance from JTP. All authors took part in writing the final version of the manuscript and approved its submission.

\section{Funding}

HL was funded by the Lundbeck Foundation, the Novo Nordisk Foundation, the Velux Foundations, and Helga og Peter Kornings Fond. CW was funded by Novo Nordisk Foundation.

\section{Availability of data and materials}

The 232 source videos used for Eulerian video magnification in this study are available on Dryad Digital Repository in either MP4 or MOV format. To access source videos, the zipped file container is downloaded from https:// datadryad.org/stash/dataset/doi:10.5061/dryad.s7h44j12q [67] and unzipped. The file name of each source video file is structured according to the following hierarchy: 1_2_3_4_5_6_7_8 where numbers refer to 1: Species name; 2: Animal\#; 3: Animal strain if relevant; 4: Experiment specific information 1; 5: Experiment specific information 2; 6: Spatial resolution of video; 7: Temporal resolution of video; 8: Camera name, for example, (1) Amex_(2) Axolotl1_(3) Leucistic_(4) Anaesthetized_(5) LateralViewWithUltrasound_(6)1920x1080px^2_(7)30fps_(8) SamsungGlaxyS6.

\section{Ethics approval and consent to participate}

Pulse wave velocity measurements based on video recording on human subjects were presented to The National Committee on Health Research Ethics (Denmark) that evaluated the project description and decided that the projected falls under method development, and no approval was required by the committee (Inquiry 71/2017). Experiments performed on axolotls and zebrafish were approved by The National Animal Experiments Inspectorate (Denmark) (Protocol\# 2015-15-0201-00615 and Protocol\# 2019-15-020100091 respectively). Mouse breeding and anesthesia were approved by Cornell University IACUC protocol \#2008-0011. Experimental work on embryonic chicken does not require IACUC approval. Video recording of sand lizard, giraffe, and elephant did not require ethics approval.

\section{Consent for publication}

The one person whose data appears in the study has given his consent for publication (signed BioMed Central consent form).

\section{Competing interests}

The authors declare that they have no competing interests.

\section{Author details}

${ }^{1}$ Nancy E. and Peter C. Meinig School of Biomedical Engineering, Cornell University, 304 Weill Hall, Ithaca, NY 14853-7202, USA. ²Department of Clinical Medicine, Aarhus University, Palle Juul-Jensens Boulevard 99, 8200 Aarhus N, Denmark. ${ }^{3}$ California State University, 333 S Twin Oaks Valley Rd, San Marcos, CA 92096, USA. ${ }^{4}$ Cornell Lab of Ornithology, Cornell University, 159 Sapsucker Woods Road, Ithaca, NY 14850, USA. ${ }^{5}$ Center for Zoo and Wild Animal Health, Copenhagen Zoo, Roskildevej 32, 2000 Frederiksberg, Denmark. ${ }^{6}$ Department of Veterinary Clinical Sciences, Faculty of Health and Medical Sciences, University of Copenhagen, Dyrlægevej 6, 1870 Frederiksberg C, Denmark. ${ }^{7}$ Department of Bioscience, Aarhus University, C.F. Møllers Allé 3, 8000 Aarhus C, Denmark.

\section{Received: 11 July 2019 Accepted: 30 October 2019}

\section{References}

1. Spallanzani L, Vassalli AM. Lettere sopra il sospetto di un nuovo senso nei pipistrelli. Torino: Nella Stamperia Reale; 1794.

2. Pierce GW, Griffin DR. Experimental determination of supersonic notes emitted by bats. J Mammal. 1938;19:454-5.

3. Griffin DR, Galambos R. The sensory basis of obstacle avoidance by flying bats. J Exp Zool. 1941:86:481-506.

4. Schevill WE, McBride AF. Evidence for echolocation by cetaceans. Deep-Sea Res. 1953;3:153-4.

5. Lissmann HW. On the function and evolution of electric organs in fish. J Exp Biol. 1958;35:156-91

6. Murray RW. Electrical sensitivity of the ampullae of Lorenzini. Nature. 1960; 187:957.

7. Scheich $H$, Langner G, Tidemann C, Coles RB, Guppy A. Electroreception and electrolocation in platypus. Nature. 1986;319:401-2.

8. Wiltschko W, Wiltschko R. Magnetic compass of European robins. Science. 1972;176(4030):62-4

9. Johnsen S, Lohmann KJ. The physics and neurobiology of magnetoreception. Nat Rev Neurosci. 2005;6:703-12.

10. Wu HY, Rubinstein M, Shih E, Guttag J, Durand F, Freeman W. Eulerian video magnification for revealing subtle changes in the world. ACM T Graphic. 2012;31:4

11. Poh MZ, McDuff DJ, Picard RW. Non-contact, automated cardiac pulse measurements using video imaging and blind source separation. Opt Express. 2010;18:10762-74

12. Liu C, Torralba A, Freeman WT, Durand F, Andelson EH. Motion magnification. ACM T Graphic. 2005;24:519-26.

13. Wang J, Drucker SM, Agrawala M, Cohen MF. The cartoon animation filter ACM T Graphic. 2006;25:1169-73.

14. Fick A. Über die Messung des Blutquantums in den Herzventrikeln. Sitz Physik Med Ges. 1870;2:16-28.

15. Green JA. The heart rate method for estimating metabolic rate: review and recommendations. Comp Biochem Physiol A Mol Integr Physiol. 2011;158: 287-304.

16. Kiceniuk JW, Jones DR. The oxygen transport system in trout (Salmo gairdneri) during sustained exercise. J Exp Biol. 1977:69:247-60.

17. Thorarensen H, Gallaugher PE, Farrell AP. The limitations of heart rate as a predictor of metabolic rate in fish. J Fish Biol. 1996;49:226-36.

18. Altimiras J, Larsen E. Non-invasive recording of heart rate and ventilation rate in rainbow trout during rest and swimming. Fish go wireless! J Fish Biol. 2000:57:197-209.

19. Wang $T$, Joyce $W$, Hicks JW. Similitude in the cardiorespiratory responses to exercise across vertebrates. Curr Opin Physiol. 2019:10:137-45.

20. Van der Hoop JM, Fahlman A, Hurst T, Rocho-Levine J, Shorter KA, Petrov V, Moore MJ. Bottlenose dolphins modify behavior to reduce metabolic effect of tag attachment. J Exp Biol. 2014;217:4229-36.

21. Armstrong JB, Malacinski GM. Developmental biology of the axolotl. New York: Oxford University Press; 1989. 
22. Gresens J. An introduction to the Mexican axolotl (Ambystoma mexicanum). Lab Anim (NY). 2004;33:41-7.

23. Roy S, Gatien S. Regeneration in axolotls: a model to aim for! Exp Gerontol. 2008;43:968-73.

24. Thygesen MM, Rasmussen MM, Madsen JG, Pedersen M, Lauridsen $H$. Propofol (2,6-diisopropylphenol) is an applicable immersion anesthetic in the axolotl with potential uses in hemodynamic and neurophysiological experiments. Regeneration. 2017;4(3):124-31.

25. Korn MJ, Cramer KS. Windowing chicken eggs for developmental studies. J Vis Exp. 2007:8:306

26. Yalcin HC, Shekhar A, Rane AA, Butcher JT. An ex-ovo chicken embryo culture system suitable for imaging and microsurgery applications. JoVE. 2010;44. http://www.jove.com/details.php?id=2154 https://doi.org/10.3791/2154.

27. Fujii S, Hirota A, Kamino K. Optical indications of pace-maker potential and rhythm generation in early embryonic chick heart. J Physiol. 1981;312:253-63.

28. Hogers B, DeRuiter MC, Baasten AM, Gittenberger-de Groot AC, Poelmann RE. Intracardiac blood flow patterns related to the yolk sac circulation of the chick embryo. Circ Res. 1995;76(5):871-7.

29. Martinsen BJ. Reference guide to the stages of chick heart embryology. Dev Dyn. 2005;233(4):1217-37.

30. Lindsey SE, Butcher JT, Yalcin HC. Mechanical regulation of cardiac development. Front Physiol. 2014;5:318.

31. Aubakir B, Nurimbetov B, Tursynbek I, Varol HA. "Vital sign monitoring utilizing Eulerian video magnification and thermography," 2016 38th Annual International Conference of the IEEE Engineering in Medicine and Biology Society (EMBC), Orlando, FL, 2016, pp. 3527-3530. https:// doi.org/10.1109/EMBC.2016.7591489.

32. Alam S, Singh SPN, Abeyratne U. "Considerations of handheld respiratory rate estimation via a stabilized Video Magnification approach," 2017 39th Annual International Conference of the IEEE Engineering in Medicine and Biology Society (EMBC), Seogwipo; 2017, pp. 4293-6. https://doi.org/10.1109/EMBC.2017.8037805

33. Ordóñez C, Cabo C, Menéndez A, Bello A. Detection of human vital signs in hazardous environments by means of video magnification. PLoS One. 2018;13(4):e0195290.

34. Al-Naji A, Perera AG, Chahl J. Remote monitoring of cardiorespiratory signals from a hovering unmanned aerial vehicle. Biomed Eng Online. 2017;16(1):101.

35. Van Hillegondsberg L, Carr J, Brey N, Henning F. Using Eulerian video magnification to enhance detection of fasciculations in people with amyotrophic lateral sclerosis. Muscle Nerve. 2017;56(6):1063-7.

36. Perrot V, Salles S, Vray D, Liebgott H. Video magnification applied in ultrasound. IEEE Trans Biomed Eng. 2018. https://doi.org/10.1109/TBME.2018. 2820384 [Epub ahead of print].

37. Barrionuevo WR, Burggren WW. $\mathrm{O}_{2}$ consumption and heart rate in developing zebrafish (Danio rerio): influence of temperature and ambient O . Am J Phys. 1999;276(2):R505-13.

38. Van Citters RL, Franklin DL, Vatner SF, Patrick T, Warren JV. Cerebral hemodynamics in the giraffe. Trans Assoc Am Phys. 1969:82:293-304.

39. Brøndum E, Hasenkam JM, Secher NH, Bertelsen MF, Grøndahl C, Petersen KK Buhl R, Aalkjaer C, Baandrup U, Nygaard H, Smerup M, Stegmann F, Sloth E, Ostergaard KH, Nissen P, Runge M, Pitsillides K, Wang T. Jugular venous pooling during lowering of the head affects blood pressure of the anesthetized giraffe. Am J Physiol Regul Integr Comp Physiol. 2009;297(4):R1058-65.

40. Smerup M, Damkjær M, Brøndum E, Baandrup UT, Kristiansen SB, Nygaard H, Funder J, Aalkjær C, Sauer C, Buchanan R, Bertelsen MF, Østergaard K Grøndahl C, Candy G, Hasenkam JM, Secher NH, Bie P, Wang T. The thick left ventricular wall of the giraffe heart normalises wall tension, but limits stroke volume and cardiac output. J Exp Biol. 2016;219(Pt 3):457-63.

41. Rabben SI, Stergiopulos N, Hellevik LR, Smiseth OA, Slørdahl S, Urheim S, Angelsen $B$. An ultrasound-based method for determining pulse wave velocity in superficial arteries. J Biomech. 2004;37:1615-22.

42. Sørensen G, Jensen JB, Udesen J, Holfort IK, Jensen JA. Pulse wave velocity in the carotid artery. IEEE International Ultrasonics Symposium Proceedings 2008 DOlhttps://doi.org/10.1109/ULTSYM.2008.0336.

43. Gesche H, Grosskurth D, Küchler G, Pazak A. Continuous blood pressure measurement by using the pulse transit time: comparison to a cuff-based method. Eur J Appl Physiol. 2011;112:309-15.

44. Carós JMS. Continuous non-invasive blood pressure estimation. Diss ETH No 20093, 2011
45. He X, Goubran RA, Liu XP. Using Eulerian video magnification framework to measure pulse transit time, IEEE ns (MeMeA), Lisboa, 2014, pp. 1-4 doi: https://doi.org/10.1109/MeMeA.2014.6860029. doi: https://doi.org/10.1109/ MeMeA.2014.6860029.

46. Shi L, Goenezen S, Haller S, Hinds MT, Thornburg KL, Rugonyi S. Alterations in pulse wave propagation reflect the degree of outflow tract banding in $\mathrm{HH} 18$ chicken embryos. Am J Physiol Heart Circ Physiol. 2013;305(3):H386-96.

47. Mellinger DK, Clark CW. Blue whale (Balaenoptera musculus) sounds from the North Atlantic. J Acoust Soc Am. 2003:114(2):1108-19.

48. Jones G. Scaling of echolocation call parameters in bats. J Exp Biol. 1999; 202(Pt 23):3359-67.

49. Lasiewski RC, Lasiewski RJ. Physiological responses of the blue-throated and Rivoli's hummingbirds. Auk. 1967;84(1):34-48.

50. Jürgens KD, Fons R, Peters $T$, Sender $S$. Heart and respiratory rates and their significance for convective oxygen transport rates in the smallest mammal, the Etruscan shrew Suncus etruscus. J Exp Biol. 1996;199(Pt 12):2579-84.

51. Payne KB, Langbauer WR, Thomas EM. Infrasonic calls of the Asian elephant (Elephas maximus). Behav Ecol Sociobiol. 1986;18:297-301.

52. Poole JH, Payne K, Langbauer WR Jr, Moss CJ. The social contexts of some very low frequency calls of African elephants. Behav Ecol Sociobiol. 1988;22: 385-92.

53. Thompson M. African forest elephant (Loxodonta africana cyclotis) vocal behavior and its use in conservation. PhD dissertation 2009. Cornell University, Ithaca, New York

54. Hedwig D, DeBellis M, Wrege PH. Not so far: attenuation of low-frequency vocalizations in a rainforest environment suggests limited acoustic mediation of social interaction in African forest elephants. Behav Ecol Sociobiol. 2018;72:33.

55. Baotic A, Stoeger AS. Sexual dimorphism in African elephant social rumbles. PloS One. 2017;12:e0177411.

56. Stoeger AS, Zeppelzauer M, Baotic A. Age-group estimation in free-ranging African elephants based on acoustic cues of low-frequency rumbles. Bioacoustics. 2014:23:231-46.

57. Stoeger AS, Baotic A. Information content and acoustic structure of male African elephant social rumbles. Sci Rep. 2016;6:27585.

58. Soltis J, Leighty KA, Wesolek CM, Savage A. The expression of affect in African elephant (Loxodonta africana) rumble vocalizations. J Comp Psychol. 2009;123:222-5.

59. Wesolek CM, Soltis J, Leighty KA, Savage A. 2009. Infant African elephant rumble vocalizations vary according to social interactions with adult females. Bioacoustics. 2009;18:227-39.

60. Herbst CT, Stoeger AS, Frey R, Lohscheller J, Titze IR, Gumpenberger M, Fitch WT. How low can you go? Physical production mechanism of elephant infrasonic vocalizations. Science. 2012;337(6094):595-9.

61. Payne KB, Thompson M, Kramer L. Elephant calling patterns as indicators of group size and composition: the basis for an acoustic monitoring system. Afr J Ecol. 2003;41:99-107.

62. Thompson ME, Schwager SJ, Payne KB, Turkalo AK. Acoustic estimation of wildlife abundance: methodology for vocal animals in forested habitats. Afr J Ecol. 2010:48:654-61.

63. Locke B. Sand lizard. 2015. https://www.flickr.com/photos/frotcott/173925 78611/in/photolist-9ypYms-nHu7fj-8rz5dw-7ScCay-aU5GAz-suVvF2-dFkJz68sUSEp-k9THCw-nRHpjd-k9Xv5P-AJ1DSo-biA6QH-k7YWpV-xByDK7-xTgigGxTkdbd-6fA7yf-gw5nAo-6pdLFi-e98xVF-7hRWBA-8LC1eN-6fwgBx-fAWyah-6 g23KY.

64. AliasAnimo. Giraffe spreading legs to get drink of water. 2014. https://www. youtube.com/watch?v=-QqQDrAhksg. Accessed 28 Dec 2015.

65. Siggraph. Eulerian video magnification for revealing subtle changes in the world. Code and Binaries. 2012. http://people.csail.mit.edu/mrub/evm/ \#code. Accessed 27 Dec 2015.

66. Quanta Research Institute, Quanta Computer Inc. LambdaVue. https:// lambda.qrilab.com/site/. Accessed 27 Dec 2015.

67. Lauridsen H, Gonzales S, Hedwig D, Perrin KL, Williams CJA, Wrege PH, et al. Extracting physiological information in experimental biology via Eulerian video magnification, v2, Dryad, Dataset. 2019. https://doi.org/10.5061/dryad. s7h44j12q. Accessed 28 Oct 2019.

\section{Publisher's Note}

Springer Nature remains neutral with regard to jurisdictional claims in published maps and institutional affiliations. 